\title{
Spectral Decomposition of the Tent Map with Varying Height
}

\author{
Suresh Subbiah ${ }^{1}$ and Dean J. Driebe ${ }^{1,2}$ \\ ${ }^{1}$ Center for Studies in Statistical Mechanics and Complex Systems \\ The University of Texas at Austin \\ Austin, Texas 78712 USA \\ ${ }^{2}$ International Solvay Institutes for Physics and Chemistry \\ 1050 Brussels, Belgium
}

\begin{abstract}
The generalized spectral decomposition of the Frobenius-Perron operator of the tent map with varying height is determined at the band-splitting points. The decomposition includes both decay onto the attracting set and the approach to the asymptotically periodic state on the attractor. Explicit compact expressions for the polynomial eigenstates are obtained using algebraic techniques.
\end{abstract}

\section{Introduction}

A natural description of the dynamics of chaotic systems is in terms of evolving probability densities [1]. On this level the time evolution in maps is governed by the linear FrobeniusPerron operator and the dynamical problem is solved by the determination of the spectral decomposition of this operator. In recent years, several authors have constructed complete and explicit spectral decompositions of the Frobenius-Perron operator of a variety of model systems [2, 3]. The most useful decompositions contain in their spectrum the decay rates characterizing the approach to equilibrium of the system. For one-dimensional piecewiselinear Markov maps such decompositions are constructed in function spaces spanned by polynomials. The dual space of these polynomials are generalized function spaces and so the decompositions are known as generalized spectral decompositions [4]. 
Chaotic systems often contain a control parameter that characterizes the strength of the chaos. A simple model system with such a control parameter is the well-known tent map with varying height [5]. The tent map with height $h$ on the unit interval is given by

$$
\mathrm{T}(x)= \begin{cases}\alpha x & 0 \leq x<\frac{1}{2} \\ \alpha(1-x) & \frac{1}{2} \leq x<1\end{cases}
$$

where the parameter $\alpha \equiv 2 h$. In Figure 1 the map (1) with height $\sqrt{2} / 2$ is shown. Note that the map acts on the unit interval $[0,1)$ but has images on $[0, h]$. As characterized

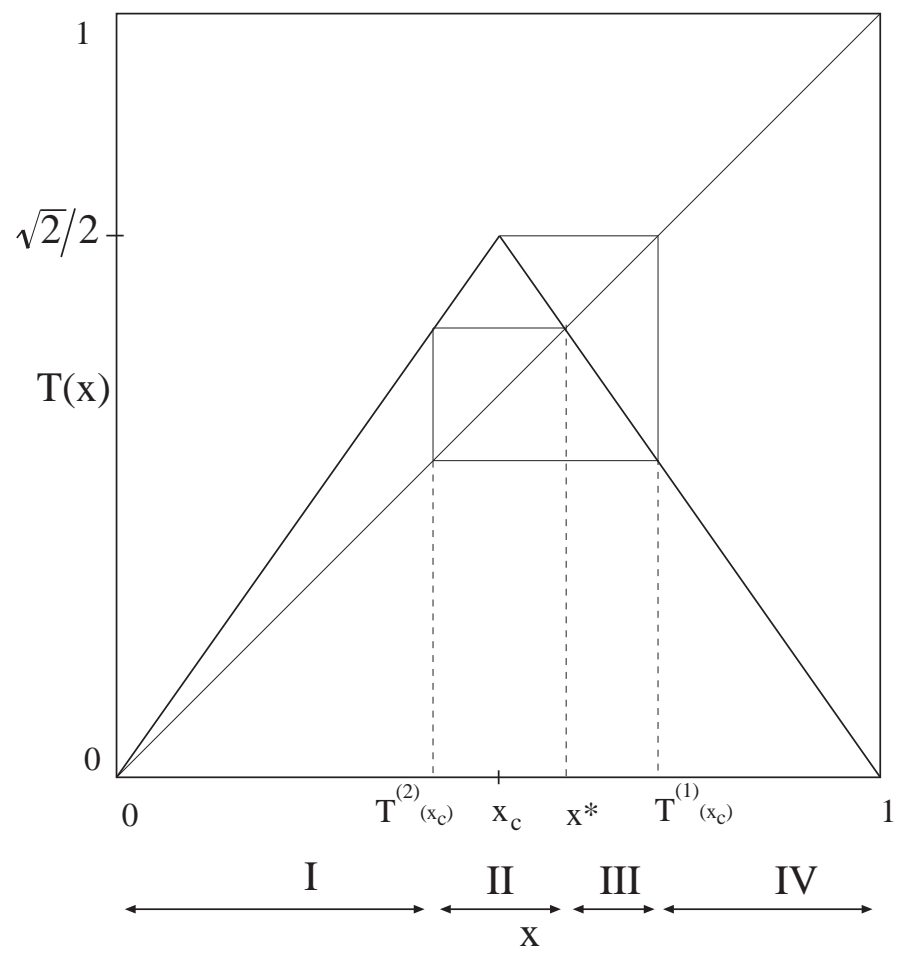

Figure 1: The tent map at $\alpha=\sqrt{2}$. For this value of $\alpha$ iterates of the critical point define four intervals around which the dynamics is organized, as discussed in Section 2.

by its Lyapunov exponent, $\log \alpha$, the map (1) switches abruptly chaotic as the height is raised past $1 / 2$. We are interested in the chaotic regime where $1 / 2<h \leq 1$, i.e., $1<$ $\alpha \leq 2$. Similar to the well-known universality of the quadratic map, it has recently been 
reported [6] that the tent map also governs the low-dimensional behavior of a wide class of nonlinear phenomena. Specifically, it was found that the dynamics of the GinzburgLandau equation, in its description of the modulational instability of a wave train, is reducible to the tent map. Under this reduction, varying the height in (四) corresponds to varying the wavelength of the initial modulational instability.

Our interest is in the statistical properties of the iterates of the tent map and in evolving probability densities. The Frobenius-Perron operator, $U$, corresponding to a map, $\mathrm{S}(x)$, defined on the unit interval evolves a probability density, $\rho(x, t)$ by one time step as

$$
\rho(x, t+1)=U \rho(x, t) \equiv \int_{0}^{1} d x^{\prime} \delta\left(x-\mathrm{S}\left(x^{\prime}\right)\right) \rho\left(x^{\prime}, t\right) .
$$

Evaluating the integral gives a sum of contributions from the inverse branches of $\mathrm{S}(x)$. The Frobenius-Perron operator corresponding to the map (1) acts explicitly on a density $\rho(x)$ as

$$
U_{\mathrm{T}} \rho(x)=\frac{1}{\alpha}\left[\rho\left(\frac{x}{\alpha}\right)+\rho\left(\frac{\alpha-x}{\alpha}\right)\right] \Theta\left(\frac{\alpha}{2}-x\right)
$$

where

$$
\Theta(a-x)= \begin{cases}1 & x \leq a \\ 0 & x>a\end{cases}
$$

The step function appears here because the map has no inverse images for $x>\alpha / 2$. For $\alpha=2$ the map has images on the whole unit interval. For this value of $\alpha$ the invariant density (being the stationary solution of (3)) is uniform on the whole unit interval. As $\alpha$ is lowered the invariant density is supported only on a subset of the interval $[\alpha(1-$ $\alpha / 2), \alpha / 2$ ] , i.e., from the second iterate to the first iterate of the critical point $x_{c} \equiv 1 / 2$. The invariant density is discontinuous at all values of the trajectory of the critical point. If the critical trajectory is periodic (or eventually periodic) there will be a finite number of discontinuities.

For $\alpha \geq \sqrt{2}$ the invariant density has nonvanishing support on all of $[\alpha(1-\alpha / 2), \alpha / 2]$. As $\alpha$ is decreased past this critical value, $\alpha_{1}$, the invariant density breaks up into two bands with a gap in the middle. Decreasing $\alpha$ past $\alpha_{2}=2^{1 / 4}$ causes the invariant density to break up into 4 bands. In general the invariant density has $2^{n}$ bands as $\alpha$ is decreased past 
$\alpha_{n}=2^{2^{-n}}$. These values of $\alpha$ are called the band-splitting points [7]. This is illustrated in Figure 2. At the band-splitting points the critical trajectory is eventually periodic
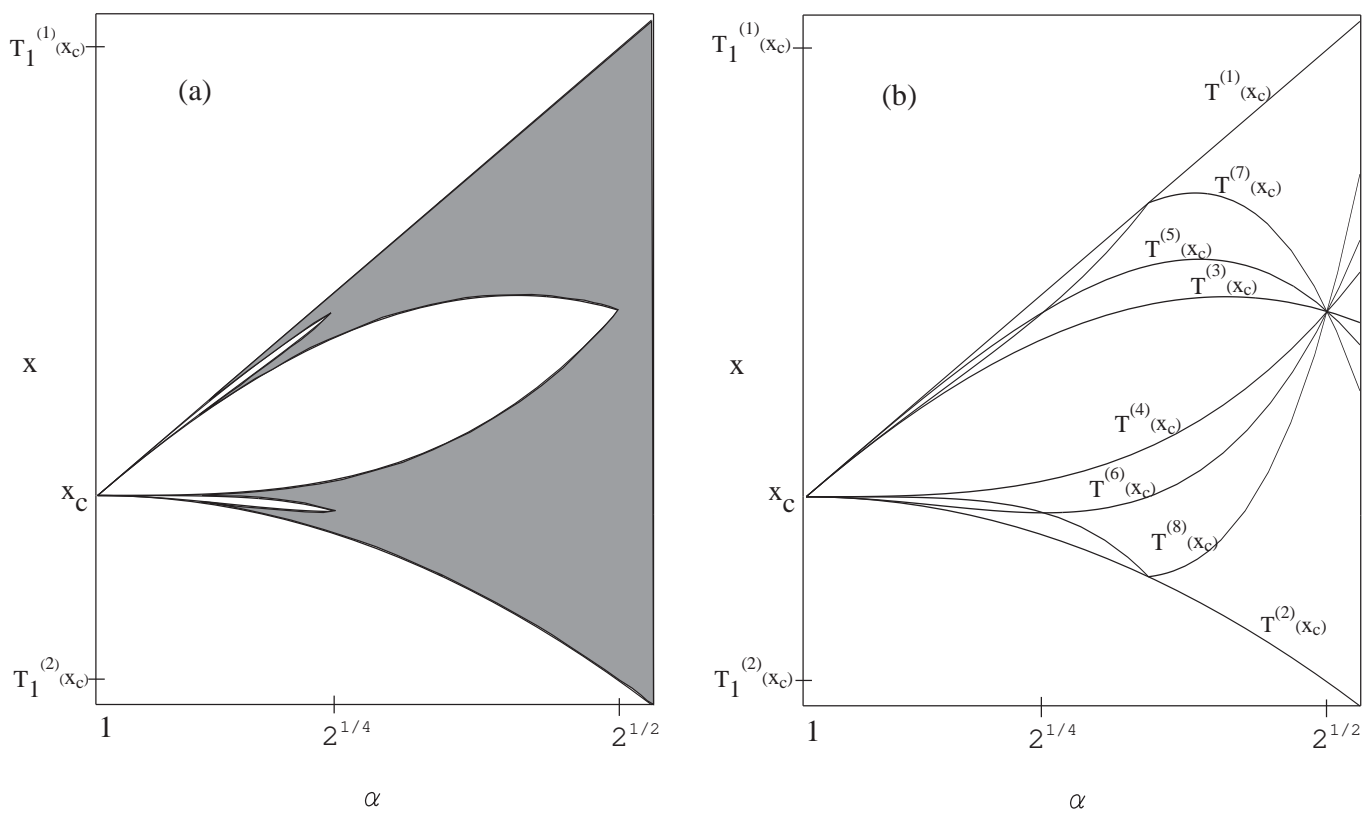

Figure 2: Bifurcation plot of the tent map with varying height showing the formation of bands as the height, parametrized by $\alpha=2 h$ on the horizontal axis, is lowered. The vertical axis is the subset $[\alpha(1-\alpha / 2), \alpha / 2]$ of the one-dimensional phase space. In (a) the shaded regions indicate where the invariant density has nonvanishing support. At this resolution we see up to the formation of the four bands at $\alpha=2^{1 / 4}$ but higher band-splitting points are not resolved. In (b) iterates of the critical trajectory as a function of $\alpha$ are plotted. These are seen to determine the band structure in (a).

and the invariant density is constant in each band and thus piecewise-constant over the unit interval. But a general initial density will also have persistent oscillating components among the bands. This feature is known as asymptotic periodicity [1].

We want to determine the spectral decomposition of the Frobenius-Perron operator so that we may expand a density or correlation function in terms of its eigenmodes. In order to do this we need the dual states or left eigenstates of $U$. These correspond to the right eigenstates of the adjoint of $U$, which is known as the Koopman operator [1]. The 
Koopman operator, $K=U^{\dagger}$, acts on a phase space function $A(x)$ as

$$
K A(x)=A(\mathrm{~T}(x))
$$

where $\mathrm{T}(x)$ is the rule for the map, such as (11). For decompositions of one-dimensional, chaotic, Markov maps in spaces spanned by polynomials the Koopman operator has eigenstates that are generalized functions or eigenfunctionals [2, 3, 4].

The decay of the $x$-autocorrelation function for the tent map at the band-splitting points and at values of $\alpha$ close to band-splitting points was calculated in [8]. The tent map has also been studied by Dorfle 9] at arbitrary values of $\alpha$ in several function spaces but he does not provide explicit complete spectral decompositions. The asymptotic periodicity of the system has been studied by Provatas and Mackey [10]. Since as $t \rightarrow \infty$ the density is supported only within $[\alpha(1-\alpha / 2), \alpha / 2]$ all these authors have only considered the map in that region and neglected transient behavior onto it. That is sufficient if one only considers the behavior of time correlation functions; but for the general evolution of densities and observables in a nonequilibrium statistical mechanics context this transient behavior must not be neglected since, as will be seen, the slowest decay modes originate from this part of the dynamics.

In the next section we construct the spectral decomposition of the Frobenius-Perron operator at the first band-splitting point. In Section 3 the decomposition at all the bandsplitting points is constructed using the self-similarity of the map at higher band-splitting points to the map at lower band-splitting points.

\section{The first band-splitting point}

From (1) the map at the first band-splitting point corresponding to the height $h=\sqrt{2} / 2$ or $\alpha=\sqrt{2}$ is

$$
\mathrm{T}_{1}(x)= \begin{cases}\sqrt{2} x & 0 \leq x<\frac{1}{2} \\ \sqrt{2}(1-x) & \frac{1}{2} \leq x<1,\end{cases}
$$

which is shown in Figure 1. The dynamics is organized around four intervals determined by the trajectory of the critical point. At the third iteration the critical trajectory settles onto 
the fixed point, $x^{*}=2-\sqrt{2}$. The four intervals: $\mathrm{I}=\left[0, \mathrm{~T}_{1}^{(2)}\left(x_{\mathrm{c}}\right)\right), \mathrm{II}=\left[\mathrm{T}_{1}^{(2)}\left(x_{\mathrm{c}}\right), \mathrm{T}_{1}^{(3)}\left(x_{\mathrm{c}}\right)\right)$ $\mathrm{III}=\left[\mathrm{T}_{1}^{(3)}\left(x_{\mathrm{c}}\right), \mathrm{T}_{1}^{(1)}\left(x_{\mathrm{c}}\right)\right)$ and $\mathrm{IV}=\left[\mathrm{T}_{1}^{(1)}\left(x_{\mathrm{c}}\right), 1\right)$, define a minimal Markov partition for the map. (These intervals are indicated in Figure 1.) Any point in the interior of interval IV is mapped onto some point in interval I in one iteration. Under successive iterations all points in the interior of I are eventually mapped into II. Any point in interval II maps into interval III in one iteration and any point in interval III maps into II in one iteration. Thus the union of the intervals II and III form the attracting set $\Omega$.

The Frobenius-Perron operator for the map (6) acts on a density as

$$
U_{\mathrm{T}_{1}} \rho(x)=\frac{1}{\sqrt{2}}\left[\rho\left(\frac{x}{\sqrt{2}}\right)+\rho\left(\frac{\sqrt{2}-x}{\sqrt{2}}\right)\right] \Theta\left(\frac{\sqrt{2}}{2}-x\right) .
$$

A general initial density continuous over the unit interval develops discontinuities at the endpoints of the four intervals described above. We thus choose a function space to consider $U_{\mathrm{T}_{1}}$ in as the space of piecewise-polynomial functions where the pieces are the four intervals described above. The invariant density has support only in $\Omega$.

The fact that points oscillate between the intervals II and III means that a general density will have a persistent oscillating component between these two intervals under time evolution. This property we will sometimes refer to in the present context as the "flip property", since the part of the density in II will all be in III (with stretching) in the next time step and vice-versa. The parts of the initial density with support in the intervals I and IV will decay onto $\Omega$. Since we will use the eigenfunctions on $\Omega$ to determine those on its complement, we first consider the decomposition on $\Omega$.

\subsection{Evolution on the attractor}

For convenience $\Omega$ is stretched onto the interval $[0,1)$. At the end of the computation we will rescale all results back to $\Omega$. The linear function that makes the stretch is

$$
\phi(x)=\left(2 / x^{*}\right) x-\sqrt{2},
$$

where $\mathrm{T}_{1}^{(2)}\left(x_{\mathrm{c}}\right) \leq x<\mathrm{T}_{1}^{(1)}\left(x_{\mathrm{c}}\right)$. The transformation (8) is a homeomorphism so that it begets a new map $R_{1}$ topologically conjugate to the part of $T_{1}$ on $\Omega$ as $R_{1}=\phi \circ T_{1} \circ \phi^{-1}$ 
given by

$$
\mathrm{R}_{1}(x)= \begin{cases}\sqrt{2} x+x^{*} & 0 \leq x<x^{*} / 2 \\ \sqrt{2}(1-x) & x^{*} / 2 \leq x<1\end{cases}
$$

where $x^{*}=2-\sqrt{2}$ is the fixed point of the map $\mathrm{R}_{1}(x)$, which is the same as the fixed point of the map $\mathrm{T}_{1}(x)$. Under this transformation, the intervals II and III are stretched to the intervals $\mathrm{A} \equiv\left[0, x^{*}\right)$ and $\mathrm{B} \equiv\left[x^{*}, 1\right)$ respectively. The map $\mathrm{R}_{1}(x)$ is shown in Figure 3 .

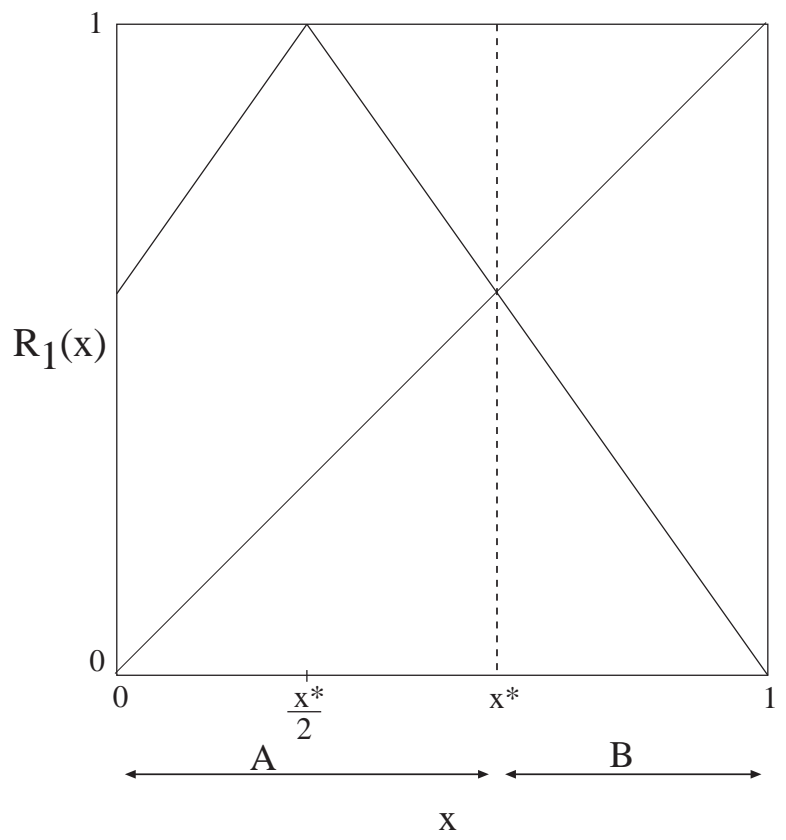

Figure 3: The rescaled Tent map at the first band-splitting point.

The Frobenius-Perron operator corresponding to the rescaled map $\mathrm{R}_{1}$ acts on a density as

$$
U_{\mathrm{R}_{1}} \rho(x)=\frac{1}{\sqrt{2}}\left[\rho\left(\frac{\sqrt{2}-x}{\sqrt{2}}\right)+\rho\left(\frac{x-x^{*}}{\sqrt{2}}\right) \Theta\left(x-x^{*}\right)\right] .
$$

The flip property of $T_{1}$ is inherited by $R_{1}$ in that the inverse image of $A$ is $B$ and viceversa. This suggests that a simpler analysis will be obtained by considering the map 
corresponding to two iterations of $R_{1}$. This map, $G_{1} \equiv R_{1} \circ R_{1}$, is given by

$$
\mathrm{G}_{1}(x)=\left\{\begin{array}{lc}
-2 x+x^{*} & 0 \leq x<x^{*} / 2 \\
2 x-x^{*} & x^{*} / 2 \leq x<\left(1+x^{*}\right) / 2 \\
-2 x+\left(2+x^{*}\right) & \left(1+x^{*}\right) / 2 \leq x<1 .
\end{array}\right.
$$

The flip property of $\mathrm{R}_{1}$ means that $\mathrm{G}_{1}$ is metrically decomposable into two independent maps on the intervals A and B, as is clear from Figure 4. From now on in this section we shall drop the subscript 1 on the maps $R_{1}$ and $G_{1}$; it being understood that we are referring to these maps at the first band-splitting point.

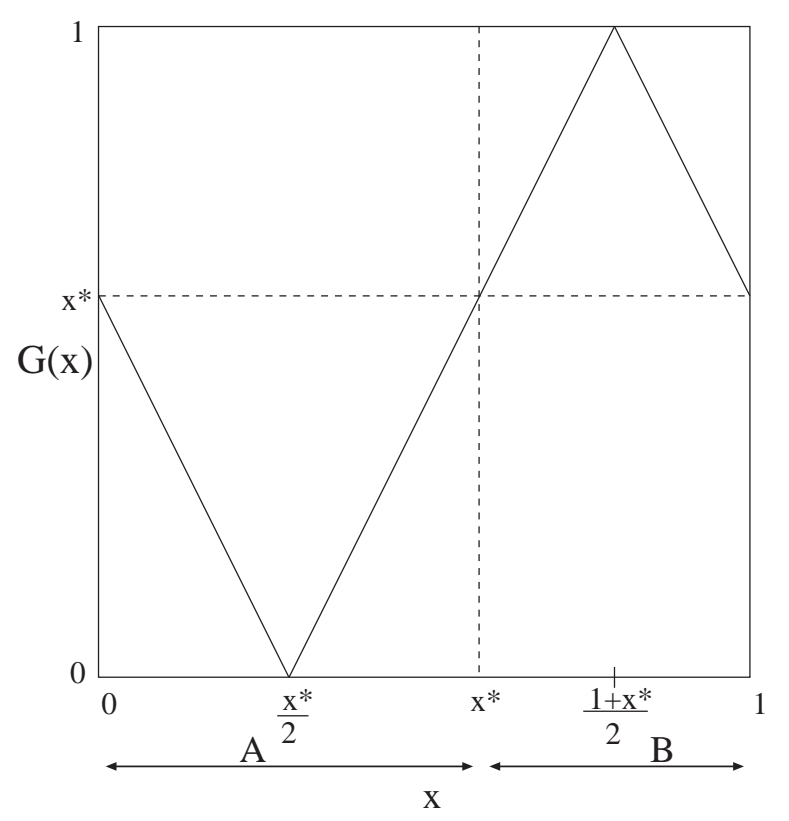

Figure 4: The map $\mathrm{G}_{1}=\mathrm{R}_{1} \circ \mathrm{R}_{1}$ is metrically decomposable into two parts each conjugate to the tent map with unit height.

The map $\mathrm{G}$ restricted to $\mathrm{A}$ is just a rescaling (with a flip) of the tent map with full height, $\mathrm{T}_{0}$, i.e., the map (1) with $\alpha=2$. This is expressed in terms of a topological conjugacy as

$$
\mathrm{G}_{\mathrm{A}}(x)=\phi_{\mathrm{A}}^{-1}(x) \circ \mathrm{T}_{0}(x) \circ \phi_{\mathrm{A}}(x)
$$


where the conjugating function $\phi_{\mathrm{A}}(x)$ is

$$
\phi_{\mathrm{A}}(x)=1-\frac{x}{x^{*}}
$$

and $x \in\left[0, x^{*}\right)$. Similarly the map on $\mathrm{B}$ is topologically conjugate to $\mathrm{T}_{0}$ as

$$
\mathrm{G}_{\mathrm{B}}(x)=\phi_{\mathrm{B}}^{-1}(x) \circ \mathrm{T}_{0}(x) \circ \phi_{\mathrm{B}}(x)
$$

where the conjugating function $\phi_{\mathrm{B}}(x)$ is

$$
\phi_{\mathrm{B}}(x)=\left(\sqrt{2} / x^{*}\right) x-\sqrt{2},
$$

and here $x \in\left[x^{*}, 1\right)$. These conjugacies are useful for us because the spectral decompositions of maps that are topologically conjugate are simply related, as is reviewed in Appendix A. The generalized spectral decomposition of $\mathrm{T}_{0}$ has been previously determined [11, 12] and is reviewed in Appendix B.

Following the discussion in those appendices gives the right eigenvectors of $\mathrm{G}_{\mathrm{A}}$ and $\mathrm{G}_{\mathrm{B}}$ as

$$
\begin{aligned}
\left|2^{-2 j}\right\rangle_{\mathrm{G}_{\mathrm{A}}} & =\frac{1}{x^{*}} B_{2 j}\left(\frac{x^{*}-x}{2 x^{*}}\right) \chi_{\mathrm{A}} \\
\left|0_{2 j+1}\right\rangle_{\mathrm{G}_{\mathrm{A}}} & =\frac{1}{x^{*}} E_{2 j+1}\left(\frac{x}{x^{*}}\right) \chi_{\mathrm{A}}, \\
\left|2^{-2 j}\right\rangle_{\mathrm{G}_{\mathrm{B}}} & =\frac{\sqrt{2}}{x^{*}} B_{2 j}\left(\frac{x-x^{*}}{\sqrt{2} x^{*}}\right) \chi_{\mathrm{B}} \\
\left|0_{2 j+1}\right\rangle_{\mathrm{G}_{\mathrm{B}}} & =\frac{\sqrt{2}}{x^{*}} E_{2 j+1}\left(\frac{\sqrt{2}}{x^{*}}\left(x-x^{*}\right)\right) \chi_{\mathrm{B}},
\end{aligned}
$$

where the associated eigenvalue is the argument of the ket vector with $\left|0_{2 j+1}\right\rangle$ meaning a null eigenpolynomial of degree $2 j+1$ and $\chi_{\mathrm{A}}$ and $\chi_{\mathrm{B}}$ are indicator functions on the intervals $\mathrm{A}$ and $\mathrm{B}$ respectively. Due to the metric decomposability of $\mathrm{G}$ these states are eigenstates of $U_{\mathrm{G}}$ as well. 
Similarly, the left eigenvectors of $\mathrm{G}_{\mathrm{A}}$ and $\mathrm{G}_{\mathrm{B}}$ are the generalized functions

$$
\begin{aligned}
\left\langle\left. 2^{-2 j}\right|_{\mathrm{G}_{\mathrm{A}}}\right. & =\frac{(-1)^{2 j-1}\left(2 x^{*}\right)^{2 j}}{(2 j) !}\left[\delta_{-}^{(2 j-1)}\left(x-x^{*}\right)-\delta_{+}^{(2 j-1)}(x)\right] \\
\left\langle\left. 0_{2 j+1}\right|_{\mathrm{G}_{\mathrm{A}}}\right. & =-\frac{\left(x^{*}\right)^{2 j+2}}{(2 j+1) !} \delta_{+}^{(2 j+1)}(x), \\
\left\langle\left. 2^{-2 j}\right|_{\mathrm{G}_{\mathrm{B}}}\right. & =\frac{(-1)^{2 j-1}\left(\sqrt{2} x^{*}\right)^{2 j}}{(2 j) !}\left[\delta_{-}^{(2 j-1)}(x-1)-\delta_{+}^{(2 j-1)}\left(x-x^{*}\right)\right] \\
\left\langle\left. 0_{2 j+1}\right|_{\mathrm{G}_{\mathrm{B}}}\right. & =\frac{-1}{(2 j+1) !}\left(\frac{x^{*}}{\sqrt{2}}\right)^{2 j+2} \delta_{-}^{(2 j+1)}(x-1),
\end{aligned}
$$

where the definitions of $\delta_{ \pm}$are given in Appendix B.

Since $U_{\mathrm{G}}=U_{\mathrm{R}}^{2}$ the spectrum of $U_{\mathrm{R}}$ is a subset of $\left\{0, \pm 2^{-j}\right\}$. Consider a non-zero eigenvalue, $2^{-2 j}$, of $U_{\mathrm{G}}$. There are two eigenvectors associated with this eigenvalue, each a polynomial of order $2 j$. Since the function space on which $U_{\mathrm{R}}$ acts has two basis elements for each degree $j$, i.e., an $j^{\text {th }}$ degree polynomial in A and an $j^{\text {th }}$ degree polynomial in $\mathrm{B}$, there should be either two eigenvectors or one eigenvector and one Jordan vector that are polynomials of degree $2 j$, associated with either one or both the eigenvalues $\left\{+2^{-j},-2^{-j}\right\}$. Since $U_{\mathrm{G}}$ does not have any Jordan vectors it follows that $U_{\mathrm{R}}$ doesn't either (for non-zero eigenvalues). The eigenvalues of $U_{\mathrm{R}}$ cannot be twofold degenerate since that would imply that all the eigenvectors of $U_{\mathrm{G}}$ are also eigenvectors of $U_{\mathrm{R}}$, which is impossible since $\mathrm{G}$ is metrically decomposable and $\mathrm{R}$ has the flip property. Therefore the non-zero eigenvalues of $\mathrm{R}$ are $+2^{-j}$ and $-2^{-j}$.

The eigenvectors of $U_{\mathrm{R}}$ with eigenvalue $\pm 2^{-j}$ are in the eigenspace spanned by the two eigenvectors of $U_{\mathrm{G}}$ corresponding to $+2^{-2 j}$. Thus they will be linear combinations as

$$
\left|+2^{-j}\right\rangle_{\mathrm{R}}=\frac{1}{2}\left(\left|+2^{-2 j}\right\rangle_{\mathrm{G}_{\mathrm{A}}}+c_{j}\left|+2^{-2 j}\right\rangle_{\mathrm{G}_{\mathrm{B}}}\right)
$$

and

$$
\left|-2^{-j}\right\rangle_{\mathrm{R}}=\frac{1}{2}\left(\left|+2^{-2 j}\right\rangle_{\mathrm{G}_{\mathrm{A}}}+d_{j}\left|+2^{-2 j}\right\rangle_{\mathrm{G}_{\mathrm{B}}}\right),
$$


where the coefficent of $1 / 2$ is put for convenient normalization. To determine $c_{j}$ we use that $\left|+2^{-j}\right\rangle_{\mathrm{R}}$ is an eigenvector of $U_{\mathrm{R}}$ as

$$
U_{\mathrm{R}}\left[\left|+2^{-2 j}\right\rangle_{\mathrm{G}_{\mathrm{A}}}+c_{j}\left|+2^{-2 j}\right\rangle_{\mathrm{G}_{\mathrm{B}}}\right]=2^{-j}\left[\left|+2^{-2 j}\right\rangle_{\mathrm{G}_{\mathrm{A}}}+c_{j}\left|+2^{-2 j}\right\rangle_{\mathrm{G}_{\mathrm{B}}}\right] .
$$

The flip property tells us that

$$
U_{\mathrm{R}}\left[c_{j}\left|+2^{-2 j}\right\rangle_{\mathrm{G}_{\mathrm{B}}}\right]=2^{-j}\left|+2^{-2 j}\right\rangle_{\mathrm{G}_{\mathrm{A}}}
$$

Substituting the explicit form (16a) of $\left|+2^{-2 j}\right\rangle_{\mathrm{G}_{\mathrm{A}}}$ and (17a) of $\left|+2^{-2 j}\right\rangle_{\mathrm{G}_{\mathrm{B}}}$ in (22) and solving for $c_{j}$ we find that $c_{j}=2^{-j}$. A similar analysis shows that $d_{j}=-2^{-j}$. Hence

$$
\left| \pm 2^{-j}\right\rangle_{\mathrm{R}}=\frac{1}{2 x^{*}}\left(B_{2 j}\left(\frac{x^{*}-x}{2 x^{*}}\right) \chi_{\mathrm{A}} \pm \frac{\sqrt{2}}{2^{j}} B_{2 j}\left(\frac{x-x^{*}}{\sqrt{2} x^{*}}\right) \chi_{\mathrm{B}}\right) .
$$

The invariant state, corresponding to the invariant density of $U_{\mathrm{R}}$ is

$$
|+1\rangle_{\mathrm{R}}=\frac{1}{2 x^{*}}\left(\chi_{\mathrm{A}}+\sqrt{2} \chi_{\mathrm{B}}\right) .
$$

This state carries all the probability under evolution of $U_{\mathrm{R}}$ and any density will have this component. The state

$$
|-1\rangle_{\mathrm{R}}=\frac{1}{2 x^{*}}\left(\chi_{\mathrm{A}}-\sqrt{2} \chi_{\mathrm{B}}\right)
$$

is the asymptotically periodic state. Only it and the invariant density survives as $t \rightarrow \infty$; but $|-1\rangle_{R}$, like decaying states, doesn't carry any probability. It does keep the memory though of the projection of the initial density on $|-1\rangle_{R}$, which is a special property of asymptotically periodic systems [1].

Now we consider the null space of $U_{R}$. The map $G$ has two independent null vectors (one in $\mathrm{A}$ and one in $\mathrm{B}$ ) for each odd degree. This implies that $\mathrm{R}$ can have either a corresponding $2 \times 2$ Jordan block or have 2 independent eigenvectors for each odd degree. The latter case is not possible since null vectors of $\mathrm{R}$ cannot have support in interval $\mathrm{B}$ because only one of the terms on the rhs of (10) acts on functions in B. Thus there is a $2 \times 2$ Jordan block for each odd degree associated with eigenvalue 0 . 
Consider the action of $U_{\mathrm{G}}=U_{\mathrm{R}}^{2}$ on a null state, $\left|0_{2 j+1}\right\rangle_{\mathrm{G}_{\mathrm{A}}}$, of $\mathrm{G}_{\mathrm{A}}$ as

$$
U_{\mathrm{R}}\left[U_{\mathrm{R}}\left|0_{2 j+1}\right\rangle_{\mathrm{G}_{\mathrm{A}}}\right]=0 \text {. }
$$

The function inside the square brackets has support only in $\mathrm{B}$, and $U_{\mathrm{R}}$ acting on any nonzero function with support in B cannot vanish in one iteration. Thus $\left|0_{2 j+1}\right\rangle_{\mathrm{R}}=\left|0_{2 j+1}\right\rangle_{\mathrm{G}_{\mathrm{A}}}$ is a null vector of $U_{\mathrm{R}}$ with explicit form given in (16b)

The Jordan vector, $\left|0_{J_{2 j+1}}\right\rangle_{\mathrm{R}}$, associated with this eigenvector satisfies

$$
U_{\mathrm{R}}\left|0_{J_{2 j+1}}\right\rangle_{\mathrm{R}}=\left|0_{2 j+1}\right\rangle_{\mathrm{R}}
$$

We may choose the Jordan vector to have support only in B as

$$
\left|0_{J_{2 j+1}}\right\rangle_{\mathrm{R}}=\eta_{2 j+1}\left|0_{2 j+1}\right\rangle_{\mathrm{G}_{\mathrm{B}}}
$$

where $\eta_{2 j+1}$ is a constant to be determined. To determine $\eta_{2 j+1}$ we apply $U_{\mathrm{R}}$ on (28) and use (27) and the explicit forms (16b) and (17b) (remembering that $\left|0_{2 j+1}\right\rangle_{\mathrm{R}}=\left|0_{2 j+1}\right\rangle_{\mathrm{G}_{\mathrm{A}}}$ ) to obtain $\eta_{2 j+1}=-1$.

\subsubsection{Left eigenstates of $U_{R}$}

The left eigenstates of $U_{\mathrm{R}}$ with non-zero eigenvalues may be determined by an approach similar to the one used to find the right eigenstates. The result is

$$
\begin{aligned}
\left\langle \pm\left. 2^{-j}\right|_{\mathrm{R}}=\frac{\left(2 x^{*}\right)^{2 j}}{(2 j) !}\left[\delta_{+}^{(2 j-1)}(x)-\delta_{-}^{(2 j-1)}\left(x-x^{*}\right)\right.\right. & \\
& \left. \pm \delta_{+}^{(2 j-1)}\left(x-x^{*}\right) \mp \delta_{-}^{(2 j-1)}(x-1)\right] .
\end{aligned}
$$

The left states with zero eigenvalues are like the right states identical to those of $U_{\mathrm{G}}$ (except for the factor of -1 for the dual state of the Jordan vector) as

$$
\begin{aligned}
\left\langle\left. 0_{2 j+1}\right|_{\mathrm{R}}\right. & =\left\langle\left. 0_{2 j+1}\right|_{\mathrm{G}_{\mathrm{A}}}\right. \\
\left\langle\left. 0_{J_{2 j+1}}\right|_{\mathrm{R}}\right. & =-\left\langle\left. 0_{2 j+1}\right|_{\mathrm{G}_{\mathrm{B}}} .\right.
\end{aligned}
$$

Note that $\left\langle\left. 0_{J_{2 j+1}}\right|_{\mathrm{R}}\right.$ is an eigenstate of the Koopman operator and $\left\langle\left. 0_{2 j+1}\right|_{\mathrm{R}}\right.$ is a Jordan state. 


\subsection{Decay onto the attractor}

As noted before, initial densities with support in the intervals I and/or IV will decay into $\Omega$. Consider a density with support only in IV at $t=0$. At $t=1$ the density has support only in I. Thus any eigenvector of $U_{\mathrm{T}_{1}}$ with support in IV can only have eigenvalue 0 . To determine such an eigenvector we write an ansatz for it as

$$
\left|0_{j}\right\rangle_{\mathrm{IV}}=f_{\mathrm{I}, j}(x) \chi_{\mathrm{I}}+f_{\mathrm{II}, j}(x) \chi_{\mathrm{II}}+f_{\mathrm{III}, j}(x) \chi_{\mathrm{III}}+f_{\mathrm{IV}, j}(x) \chi_{\mathrm{IV}},
$$

where the subscript IV on the ket denotes that it describes decay out of interval IV and we take $f_{\mathrm{IV}, j}(x)$ as a polynomial of order $j$. Applying $U_{\mathrm{T}_{1}}$ to (31) and collecting terms that are multiplied by the same indicator function we get

$$
\begin{aligned}
U_{\mathrm{T}_{1}}\left|0_{j}\right\rangle_{\mathrm{IV}}= & {\left[f_{\mathrm{I}, j}\left(\frac{x}{\sqrt{2}}\right)+f_{\mathrm{IV}, j}\left(1-\frac{x}{\sqrt{2}}\right)\right] \chi_{\mathrm{I}} } \\
& +\left[f_{\mathrm{I}, j}\left(\frac{x}{\sqrt{2}}\right)+f_{\mathrm{III}, j}\left(1-\frac{x}{\sqrt{2}}\right)\right] \chi_{\mathrm{II}} \\
& +\left[f_{\mathrm{II}, j}\left(\frac{x}{\sqrt{2}}\right)+f_{\mathrm{II}, j}\left(1-\frac{x}{\sqrt{2}}\right)\right] \chi_{\mathrm{III}} \cdot
\end{aligned}
$$

Since $\left|0_{j}\right\rangle_{\text {IV }}$ is a null eigenstate the coefficients of each of the indicator functions must vanish. Since $f_{\mathrm{IV}, j}$ is a polynomial of degree $j$, it follows that $f_{\mathrm{I}, j}$ and $f_{\mathrm{III}, j}$ are also polynomials of degree $j$. We choose $f_{\mathrm{II}, j}$ to be zero. Clearly, $f_{\mathrm{III}, j}=f_{\mathrm{IV}, j}$ and choosing it as $(x-1)^{j}$ fixes $f_{\mathrm{I}, j}$. We thus have

$$
\left|0_{j}\right\rangle_{\mathrm{IV}}=(-1)^{j+1} x^{j} \chi_{\mathrm{I}}+(x-1)^{j}\left(\chi_{\mathrm{III}}+\chi_{\mathrm{IV}}\right)
$$

We note that because of the degeneracy associated with eigenvalue 0 in interval IV, this choice of eigenvectors is not unique. The left eigenstates (given below) associated with this choice of right eigenvectors is unique and therefore when we expand an arbitrary density in terms of the right eigenvectors, the expansion coefficents are uniquely defined.

The action of $U_{\mathrm{T}_{1}}$ on a function with support only on $\mathrm{I}$ is given by

$$
U_{\mathrm{T}_{1}}\left[f(x) \chi_{\mathrm{I}}\right]=\frac{1}{\sqrt{2}} f(x / \sqrt{2})\left(\chi_{\mathrm{I}}+\chi_{\mathrm{II}}\right) .
$$


Acting with $U_{\mathrm{T}_{1}}$ on a monomial in I gives

$$
U_{\mathrm{T}_{1}}\left[x^{j} \chi_{\mathrm{I}}\right]=\frac{x^{j}}{(\sqrt{2})^{j+1}}\left(\chi_{\mathrm{I}}+\chi_{\mathrm{II}}\right)
$$

so that the eigenvectors of $U_{\mathrm{T}_{1}}$ with support in I are monomials in I. To determine their form in the other intervals we again write an ansatz as we did for the eigenvectors with support in IV as

$$
\left|2^{-(j+1) / 2}\right\rangle_{\mathrm{I}}=x^{j} \chi_{\mathrm{I}}+g_{\mathrm{II}, j}(x) \chi_{\mathrm{II}}+g_{\mathrm{III}, j}(x) \chi_{\mathrm{III}},
$$

where the associated eigenvalue, appearing as the argument of the ket, is seen from (35). In equation (36) and below the subscript I on a ket implies that it describes decay out of region I. Note that the $j=0$ mode here is the slowest decay mode in this system. Since $U_{\mathrm{T}_{1}}$ does not raise the degree of the polynomial it acts on, $g_{\mathrm{II}, j}(x)$ and $g_{\mathrm{III}, j}(x)$ are polynomials of degree $j$. Applying $U_{\mathrm{T}_{1}}$ to (36) and using (35) and the fact that the function on the rhs of (36) is an eigenvector with eigenvalue $2^{-(j+1) / 2}$, we find that

$$
U_{\mathrm{T}_{1}}\left(g_{\mathrm{II}, j}(x) \chi_{\mathrm{II}}+g_{\mathrm{III}, j}(x) \chi_{\mathrm{III}}\right)=2^{-(j+1) / 2}\left(g_{\mathrm{II}, j}(x) \chi_{\mathrm{II}}+g_{\mathrm{III}, j}(x) \chi_{\mathrm{III}}-x^{j} \chi_{\mathrm{II}}\right) .
$$

The determination of $g_{\mathrm{II}, j}(x)$ and $g_{\mathrm{III}, j}(x)$ is described in Appendix C

The explicit form of the first few of these eigenvectors is

$$
\begin{aligned}
\left|2^{-1 / 2}\right\rangle_{\mathrm{I}}= & \chi_{\mathrm{I}}+\frac{1}{2(1-\sqrt{2})}|1\rangle_{\Omega}+\frac{1}{2(1+\sqrt{2})}|-1\rangle_{\Omega} \\
\left|2^{-1}\right\rangle_{\mathrm{I}}= & x \chi_{\mathrm{I}}-\frac{1}{4\left(x^{*}\right)}|1\rangle_{\Omega}+\frac{1}{12}|-1\rangle_{\Omega}+\frac{\left(x^{*}\right)^{2}}{2}|0\rangle_{\Omega} \\
\left|2^{-3 / 2}\right\rangle_{\mathrm{I}}= & x^{2} \chi_{\mathrm{I}}-\frac{\sqrt{2} x^{*}}{12}|1\rangle_{\Omega}+\frac{(9 \sqrt{2}-8) x^{*}}{84}|-1\rangle_{\Omega}+\frac{\sqrt{2}\left(x^{*}\right)^{3}}{2}|0\rangle_{\Omega} \\
& -\frac{\sqrt{2}\left(x^{*}\right)^{3}}{2}\left|2^{-1}\right\rangle_{\Omega}+\frac{\left(x^{*}\right)^{4}}{2(1+\sqrt{2})}\left|-2^{-1}\right\rangle_{\Omega} .
\end{aligned}
$$

The subscript $\Omega$ on a ket implies that it is a right state of $U_{\mathrm{R}}$ which has been rescaled into the attractor, $\Omega$, of $U_{\mathrm{T}_{1}}$. The explicit form of these states is given in Table 1 . 


\subsection{Left eigenstates of $U_{\mathrm{T}_{1}}$}

It is easily seen that the left states given by

$$
\left\langle\left. 0_{j}\right|_{\mathrm{IV}}=\frac{(-1)^{j}}{j !} \delta_{-}^{(j)}(x-1)\right.
$$

form a bi-orthonormal set with the null states in (33). These left states are also orthogonal to all the other right states of $U_{\mathrm{T}_{1}}$. The left states associated with the transient decay states out of I are given by

$$
\left\langle\left. 2^{-(j+1) / 2}\right|_{\mathrm{I}}=\frac{1}{j !}\left[(-1)^{j} \delta_{+}^{(j)}(x)+\delta_{-}^{(j)}(x-1)\right] .\right.
$$

The left states of $U_{\mathrm{R}}$ are not the left states of $U_{\mathrm{T}_{1}}$, even though the right states of $U_{\mathrm{R}}$ are also right states of $U_{\mathrm{T}_{1}}$. This is because $U_{\mathrm{T}_{1}}$ acting on a density contained within $\Omega$ will continue to be in $\Omega$. But in general the Koopman operator, $K_{\mathrm{T}_{1}}$, acting on a function with support only in $\Omega$ will result in the function having support outside $\Omega$ too. The left states of $U_{\mathrm{R}}$ scaled back to $\Omega$ form a bi-orthonormal set with the right states contained in $\Omega$, but they are not orthogonal to the transient states that decay into $\Omega$. To make them so we use Gram-Schmidt orthogonalization. The results are given in Table 1.

\subsection{The spectral decomposition}

Using all the eigenstates and eigenvalues given in Table 1 we may write the action of $U_{\mathrm{T}_{1}}^{t}$ in terms of its spectral decomposition as

$$
\begin{aligned}
U_{\mathrm{T}_{1}}^{t}= & |1\rangle\left\langle\left. 1\right|_{\Omega}+(-1)^{t} \mid-1\right\rangle\left\langle-\left.1\right|_{\Omega}+\sum_{j=0}^{\infty}\left(2^{-(2 j+1) / 2}\right)^{t} \mid 2^{-(2 j+1) / 2}\right\rangle\left\langle\left. 2^{-(2 j+1) / 2}\right|_{\mathrm{I}}\right. \\
& +\sum_{j=1}^{\infty}\left(2^{-j}\right)^{t}\left[\left|+2^{-j}\right\rangle\left\langle+\left.2^{-j}\right|_{\Omega}+\mid 2^{-j}\right\rangle\left\langle\left. 2^{-j}\right|_{\mathrm{I}}\right]\right. \\
& +\sum_{j=1}^{\infty}\left(-2^{-j}\right)^{t}\left|-2^{-j}\right\rangle\left\langle-\left.2^{-j}\right|_{\Omega}+\sum_{j=0}^{\infty} \delta_{1, t} \mid 0_{2 j+1}\right\rangle\left\langle\left. 0_{J_{2 j+1}}\right|_{\Omega}\right. \\
& +\sum_{j=0}^{\infty} \delta_{0, t}\left[\left|0_{2 j+1}\right\rangle\left\langle\left. 0_{2 j+1}\right|_{\Omega}+\mid 0_{J_{2 j+1}}\right\rangle\left\langle\left. 0_{J_{2 j+1}}\right|_{\Omega}+\mid 0_{j}\right\rangle\left\langle\left. 0_{j}\right|_{\mathrm{IV}}\right]\right.
\end{aligned}
$$

where the subscript on the bra states also identifies their dual ket states. 


\begin{tabular}{|c|c|c|c|}
\hline eigenvalue & degeneracy & symbol & eigenvector \\
\hline 1 & 1 & $\begin{array}{l}|1\rangle_{\Omega} \\
\left\langle\left. 1\right|_{\Omega}\right.\end{array}$ & $\begin{array}{l}\chi_{\mathrm{II}}+\sqrt{2} \chi_{\mathrm{III}} \\
\frac{\left(x^{*}\right)^{2}}{2}\left(\chi_{\mathrm{II}}+\chi_{\mathrm{III}}\right) \\
-\sum_{k=0}^{\infty}\left(a_{0, k}\left\langle\left. 2^{-(k+1) / 2}\right|_{\mathrm{I}}+\alpha_{0, k}^{\prime}\left\langle\left. 0_{k}\right|_{\mathrm{IV}}\right)\right.\right.\end{array}$ \\
\hline-1 & 1 & $\begin{array}{l}|-1\rangle_{\Omega} \\
\left\langle-\left.1\right|_{\Omega}\right.\end{array}$ & $\begin{array}{l}\chi_{\mathrm{II}}-\sqrt{2} \chi_{\mathrm{III}} \\
\frac{\left(x^{*}\right)^{2}}{2}\left(\chi_{\mathrm{II}}-\chi_{\mathrm{III}}\right) \\
-\sum_{k=0}^{\infty}\left(b_{0, k}\left\langle\left. 2^{-(k+1) / 2}\right|_{\mathrm{I}}-\alpha_{0, k}^{\prime}\left\langle\left. 0_{k}\right|_{\mathrm{IV}}\right)\right.\right.\end{array}$ \\
\hline$+\frac{1}{2^{j}}$ & 1 & $\begin{array}{l}\left|+2^{-j}\right\rangle_{\Omega} \\
\left\langle+\left.2^{-j}\right|_{\Omega}\right.\end{array}$ & $\begin{array}{l}B_{2 j}\left(\frac{x^{*}-\phi(x)}{2 x^{*}}\right) \chi_{\mathrm{II}}+\frac{\sqrt{2}}{2^{j}} B_{2 j}\left(\frac{\phi(x)-x^{*}}{\sqrt{2} x^{*}}\right) \chi_{\mathrm{III}} \\
\frac{\left(x^{*}\right)^{4 j-2}}{(2 j) !}\left[\delta_{+}^{(2 j-1)}\left(x-\mathrm{T}^{(2)}\left(x_{c}\right)\right)-\delta_{-}^{(2 j-1)}\left(x-x^{*}\right)\right. \\
\left.+\delta_{+}^{(2 j-1)}\left(x-x^{*}\right)-\delta_{-}^{(2 j-1)}\left(x-\mathrm{T}^{(1)}\left(x_{c}\right)\right)\right] \\
-\sum_{k=2 j}^{\infty}\left(a_{j, k}\left\langle\left. 2^{-(k+1) / 2}\right|_{\mathrm{I}}+\alpha_{j, k}^{\prime}\left\langle\left. 0_{k}\right|_{\mathrm{IV}}\right)\right.\right.\end{array}$ \\
\hline$-\frac{1}{2^{j}}$ & 1 & $\begin{array}{l}\left|-2^{-j}\right\rangle_{\Omega} \\
\left\langle-\left.2^{-j}\right|_{\Omega}\right.\end{array}$ & $\begin{array}{l}B_{2 j}\left(\frac{x^{*}-\phi(x)}{2 x^{*}}\right) \chi_{\mathrm{II}}-\frac{\sqrt{2}}{2^{j}} B_{2 j}\left(\frac{\phi(x)-x^{*}}{\sqrt{2} x^{*}}\right) \chi_{\mathrm{III}} \\
\frac{\left(x^{*}\right)^{4 j-2}}{(2 j) !}\left[\delta_{+}^{(2 j-1)}\left(x-\mathrm{T}^{(2)}\left(x_{c}\right)\right)-\delta_{-}^{(2 j-1)}\left(x-x^{*}\right)\right. \\
\left.-\delta_{+}^{(2 j-1)}\left(x-x^{*}\right)+\delta_{-}^{(2 j-1)}\left(x-\mathrm{T}^{(1)}\left(x_{c}\right)\right)\right] \\
-\sum_{k=2 j}^{\infty}\left(b_{j, k}\left\langle\left. 2^{-(k+1) / 2}\right|_{\mathrm{I}}-\alpha_{j, k}^{\prime}\left\langle\left. 0_{k}\right|_{\mathrm{IV}}\right)\right.\right.\end{array}$ \\
\hline 0 & $\begin{array}{c}\infty \\
(2 \times 2 \\
\text { Jordan } \\
\text { blocks })\end{array}$ & $\begin{array}{l}\left|0_{2 j+1}\right\rangle_{\Omega} \\
\left\langle\left. 0_{2 j+1}\right|_{\Omega}\right. \\
\left|0_{J_{2 j+1}}\right\rangle_{\Omega} \\
\left\langle\left. 0_{J_{2 j+1}}\right|_{\Omega}\right.\end{array}$ & $\begin{array}{l}E_{2 j+1}\left(\frac{\phi(x)}{x^{*}}\right) \chi_{\mathrm{II}} \\
-\left(\frac{x^{*}}{\sqrt{2}}\right)^{4 j+2} \frac{1}{(2 j+1) !} \delta_{+}^{(2 j+1)}\left(x-\mathrm{T}^{(2)}\left(x_{c}\right)\right) \\
-\sum_{k=2 j+1}^{\infty} c_{j, k}\left\langle\left. 2^{-(k+1) / 2}\right|_{\mathrm{I}}\right. \\
-\sqrt{2}\left(E_{2 j+1}\left(\frac{\sqrt{2}}{x^{*}}\left(\phi(x)-x^{*}\right)\right)\right) \chi_{\mathrm{III}} \\
\frac{\left(x^{*}\right)^{4 j+2}}{\sqrt{2}(2 \sqrt{2})^{2 j+1}} \frac{1}{(2 j+1) !} \delta_{-}^{(2 j+1)}\left(x-\mathrm{T}^{(1)}\left(x_{c}\right)\right) \\
-\sum_{k=2 j+1}^{\infty} \gamma_{j, k}^{\prime}\left\langle\left. 0_{k}\right|_{\mathrm{IV}}\right.\end{array}$ \\
\hline$\left(\frac{1}{\sqrt{2}}\right)^{j+1}$ & 1 & $\begin{array}{l}\left|2^{-(j+1) / 2}\right\rangle_{\mathrm{I}} \\
\left\langle\left. 2^{-(j+1) / 2}\right|_{\mathrm{I}}\right.\end{array}$ & $\begin{array}{l}x^{j} \chi_{\mathrm{I}}+\sum_{i=0} a_{i, j}\left|2^{-i}\right\rangle+b_{i, j}\left|-2^{-i}\right\rangle+c_{i, j}\left|0_{i}\right\rangle \\
\frac{1}{j !}\left((-1)^{j} \delta^{(j)}(x)+\delta^{(j)}(x-1)\right)\end{array}$ \\
\hline 0 & $\infty$ & $\begin{array}{l}\left|0_{j}\right\rangle_{\mathrm{IV}} \\
\left\langle\left. 0_{j}\right|_{\mathrm{IV}}\right.\end{array}$ & $\begin{array}{l}(-1)^{j+1} x^{j} \chi_{\mathrm{I}}+(x-1)^{j}\left(\chi_{\mathrm{III}}+\chi_{\mathrm{IV}}\right) \\
\frac{(-1)^{j}}{j !} \delta^{(j)}(x-1)\end{array}$ \\
\hline
\end{tabular}

Table 1: Elements of the spectral decomposition of the tent map at the first band splitting point. The constants $a_{i, j} \equiv\left\langle\left. 2^{-i}\right|_{\Omega}(2)^{-(j+1) / 2}\right\rangle_{\mathrm{I}}, b_{i, j} \equiv\left\langle-\left.2^{-i}\right|_{\Omega}(2)^{-(j+1) / 2}\right\rangle_{\mathrm{I}}$ and $c_{i, j} \equiv\left\langle\left. 0_{i}\right|_{\Omega}(2)^{-(j+1) / 2}\right\rangle_{\mathrm{I}}$ and are given in Appendix C. The constants $\alpha_{i, j}^{\prime} \equiv\left\langle+\left.2^{-i}\right|_{\Omega} 0_{j}\right\rangle_{\mathrm{IV}}, \beta_{i, j}^{\prime} \equiv\left\langle-\left.2^{-i}\right|_{\Omega} 0_{j}\right\rangle_{\mathrm{IV}}=-\alpha_{i, j}^{\prime}$ and $\gamma_{i, j}^{\prime} \equiv\left\langle\left. 0_{J_{i}}\right|_{\Omega} 0_{j}\right\rangle_{\mathrm{IV}}$ and $\phi(x)$ is defined in (8). 


\section{Higher band-splitting points}

We can determine the spectral decomposition of the tent map at any band-splitting point (bsp) by generalizing the approach used in the previous section for the first bsp. For the rescaled map at $\alpha=\sqrt{2}$ we found that by considering its square it separated into two parts that were directly related by a simple change of scale (including a reflection for one part) to the tent map at full height where $\alpha=2$. This relationship also holds generally 10, 13] between the map at the $n^{\text {th }}$ bsp, $\left(\alpha_{n}=2^{2^{-n}}\right)$, and the $(n-1)^{\text {th }}$ bsp, $\left(\alpha_{n-1}=2^{2^{-(n-1)}}=\alpha_{n}^{2}\right)$.

The tent map at $\alpha_{n}$ on the interval $\left[\mathrm{T}_{n}^{(2)}\left(x_{c}\right), \mathrm{T}_{n}^{(1)}\left(x_{c}\right)\right)$, which contains the attractor, is first stretched to the unit interval $[0,1)$ to make the rescaled map $\mathrm{R}_{n}$. The linear function that makes this stretch is

$$
\phi_{n}(x)=\frac{2 x-\alpha_{n}\left(2-\alpha_{n}\right)}{\alpha_{n}\left(\alpha_{n}-1\right)} .
$$

Under (42) the map $\mathrm{T}_{n}$ transforms to $\mathrm{R}_{n}=\phi_{n} \circ \mathrm{T}_{n} \circ \phi_{n}^{-1}$ and is given by

$$
\mathrm{R}_{n}(x)= \begin{cases}2-\alpha_{n}(1-x) & 0 \leq x<\left(\alpha_{n}-1\right) / \alpha_{n} \\ \alpha_{n}(1-x) & \left(\alpha_{n}-1\right) / \alpha_{n} \leq x<1\end{cases}
$$

We then compose $\mathrm{R}_{n}$ with itself to obtain $\mathrm{G}_{n} \equiv \mathrm{R}_{n} \circ \mathrm{R}_{n}$. As is illustrated in Figure 5 for the $2^{\text {nd }}$ bsp, 1 t can be shown easily that in general:

(a) $\mathrm{G}_{n}(x)$ in the interval $X_{n, \mathrm{~A}} \equiv\left[0, \mathrm{R}_{n}^{(4)}\left(x_{c}\right)\right.$ ) is topologically conjugate to $\mathrm{R}_{n-1}$ (the rescaled map at higher height) in the interval $[0,1)$ as

$$
\mathrm{R}_{n-1}=\phi_{n, \mathrm{~A}} \circ \mathrm{G}_{n, \mathrm{~A}} \circ \phi_{n, \mathrm{~A}}^{-1}
$$

where

$$
\phi_{n, \mathrm{~A}}=1-\frac{x}{\alpha_{n}\left(\alpha_{n}-1\right)}, \quad x \in X_{n, \mathrm{~A}}
$$

(b) $\mathrm{G}_{n}(x)$ in the interval $X_{n, \mathrm{~B}} \equiv\left[\mathrm{R}_{n}^{(3)}\left(x_{c}\right), 1\right)$ is topologically conjugate to $\mathrm{R}_{n-1}$ in the interval $[0,1)$ as

$$
\mathrm{R}_{n-1}=\phi_{n, \mathrm{~B}} \circ \mathrm{G}_{n, \mathrm{~B}} \circ \phi_{n, \mathrm{~B}}^{-1}
$$


where

$$
\phi_{n, \mathrm{~B}}=\frac{x-\left(2-\alpha_{n}\right)}{\alpha_{n}-1}, \quad x \in X_{n, \mathrm{~B}}
$$

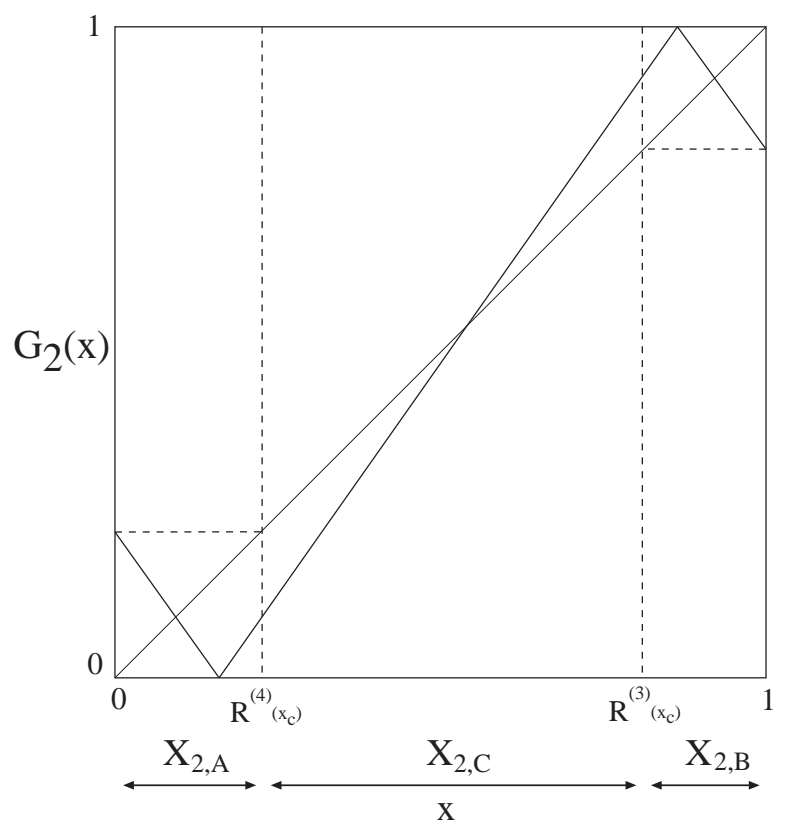

Figure 5: At the second band-splitting point, $\mathrm{G}_{2}$ is conjugate in $X_{2, \mathrm{~A}}$ and $X_{2, \mathrm{~B}}$ to $\mathrm{R}_{1}$ shown in Figure 3 . The central transient region is shown as $X_{2, \mathrm{C}}$.

At the $n^{\text {th }}$ bsp the critical trajectory is eventually periodic with period $2^{n-1}(n \geq 1)$, and therefore the number of discontinuities that appear under time evolution of an initially smooth density is finite. Equations (44) - (47) imply that the band structure at the $n^{\text {th }}$ bsp consists of 2 scaled copies of the band structure at the $(n-1)^{\text {th }}$ bsp separated by the interval $\left[\mathrm{R}_{n}^{(4)}\left(x_{c}\right), \mathrm{R}_{n}^{(3)}\left(x_{c}\right)\right) \equiv X_{n, \mathrm{C}}$, which makes one band. Therefore we have the 
following recursion relation for $S_{n}$, the number of bands at the $n^{\text {th }}$ bsp for the map $\mathrm{R}_{n}$,

$$
S_{n}=2 S_{n-1}+1
$$

for $n \geq 2$, where $S_{1}=2$. Solving this recursion relation gives

$$
S_{n}=2^{n}+2^{n-1}-1
$$

for $n \geq 1$. Of the $S_{n}$ bands, the invariant density has support only on $2^{n}$ bands. The density is transient on the other $2^{n-1}-1$ bands. The function space we consider at the $n^{\text {th }}$ bsp is piecewise polynomial, with each piece extending over one band.

\subsection{Decomposition on the attractor}

Associated with the $2^{n}$ bands that make up $\Omega_{n}$, the attractor at the $n^{\text {th }}$ bsp, are $2^{n}$ eigen/Jordan vectors of each polynomial degree $j$. We denote an element in the spectrum of $U_{\mathrm{R}_{n}}$ associated with states on $\Omega_{n}$ as $\lambda_{k, j}^{n}$ and the right eigen/Jordan vector associated with it as $\left|\lambda_{k, j}^{n}\right\rangle$. The superscript $n$ stands for the order of the band-splitting point. The index $k$ is an integer from 1 to $2^{n}$ and distinguishes between the $2^{n}$ independent eigen/Jordan vectors of degree $j$, at the $n^{\text {th }}$ bsp.

Since $\mathrm{G}_{n, \mathrm{~A}}$ and $\mathrm{G}_{n, \mathrm{~B}}$ are topologically conjugate to $\mathrm{R}_{n-1}$ all three share the same spectrum. The map $\mathrm{G}_{n}$ is the union of $\mathrm{G}_{n, \mathrm{~A}}, \mathrm{G}_{n, \mathrm{~B}}$ and the part of $\mathrm{G}_{n}$ on the central transient interval $X_{n, \mathrm{C}}$. The spectrum of $\mathrm{R}_{n}$ on $\Omega_{n}$ is determined from the spectrum of $\mathrm{G}_{n}$ on $\Omega_{n}$. Consider a non-zero eigenvalue $\lambda_{k, 2 j}^{n-1}$ of $\mathrm{R}_{n-1}$ (we will show later that as at the first bsp the right states corresponding to non-zero eigenvalues on the attractor are even-order polynomials), which is also an eigenvalue of $\mathrm{G}_{n}$ with degeneracy 2 (one for $\mathrm{G}_{n, \mathrm{~A}}$ and one for $\left.\mathrm{G}_{n, \mathrm{~B}}\right)$. Using arguments parallel to those that we used at the first bsp we deduce that $\mathrm{R}_{n}$ has two distinct eigenvalues $+\sqrt{\lambda_{k, 2 j}^{n-1}}$ and $-\sqrt{\lambda_{k, 2 j}^{n-1}}$. By induction $\mathrm{R}_{n+1}$ on $\Omega_{n+1}$ has in its spectrum the eigenvalues $+\left(\lambda_{k, 2 j}^{n-1}\right)^{1 / 4},-\left(\lambda_{k, 2 j}^{n-1}\right)^{1 / 4},+i\left(\lambda_{k, 2 j}^{n-1}\right)^{1 / 4}$ and $-i\left(\lambda_{k, 2 j}^{n-1}\right)^{1 / 4}$. Thus from the non-zero eigenvalues at the $0^{\text {th }}$ bsp we can determine the non-zero eigenvalues at the $n^{\text {th }}$ bsp by taking square roots of $2^{-2 j}$ recursively, $n$ times. 
Thus

$$
\lambda_{k, 2 j}^{n}=\left(\frac{1}{2}\right)^{2 j / 2^{n}} \exp \left(\frac{2 \pi i k}{2^{n}}\right),
$$

where $k=1,2, \ldots, 2^{n}$ and $j=0,1,2, \ldots$. Note that for $k \leq 2^{n-1}$

$$
\lambda_{k, 2 j}^{n}=-\lambda_{k+2^{n-1}, 2 j}^{n}
$$

At the $0^{\text {th }}$ bsp the eigenvalue 0 is infinitely degenerate with an associated eigenpolynomial of odd degree for each occurence of the eigenvalue. In the previous section we saw that at the $1^{\text {st }}$ bsp there are an infinite number of $2 \times 2$ Jordan blocks associated with eigenvalue zero, one each for each odd degree. We will show by explicit construction below that this trend continues and at the $n^{\text {th }}$ bsp, $\mathrm{R}_{n}$ has a $2^{n} \times 2^{n}$ Jordan block for every odd degree.

\subsubsection{Right states on the attractor}

To determine the right states on $\Omega_{n}$ with non-zero eigenvalues we begin similar to (20) by writing the right states at the $n^{\text {th }}$ bsp in terms of those at the $(n-1)^{\text {th }}$ bsp as

$$
\begin{aligned}
\left|\lambda_{k, 2 j}^{n}\right\rangle & =\left|\lambda_{k, 2 j}^{n-1}\right\rangle_{\mathrm{G}_{n, \mathrm{~A}}}+c_{k, 2 j}^{n}\left|\lambda_{k, 2 j}^{n-1}\right\rangle_{\mathrm{G}_{n, \mathrm{~B}}} \\
\left|\lambda_{k+2^{n-1}, 2 j}^{n}\right\rangle & =\left|\lambda_{k, 2 j}^{n-1}\right\rangle_{\mathrm{G}_{n, \mathrm{~A}}}+d_{k, 2 j}^{n}\left|\lambda_{k, 2 j}^{n-1}\right\rangle_{\mathrm{G}_{n, \mathrm{~B}}},
\end{aligned}
$$

where $k=1,2, \ldots, 2^{n-1}$. In equation (52) and in the remaining part of this subsection kets without any subscript denote right states of $\mathrm{R}$ at the appropriate bsp. Conjugacies (44) and (46) imply that the eigenvectors of $\mathrm{G}_{n, \mathrm{~A}}$ and $\mathrm{G}_{n, \mathrm{~B}}$ are related to those of $\mathrm{R}_{n-1}$ as

$$
\begin{aligned}
\left|\lambda_{k, 2 j}^{n-1}\right\rangle_{\mathrm{G}_{n, \mathrm{~A}}} & =U_{\phi_{n, \mathrm{~A}}^{-1}}\left|\lambda_{k, 2 j}^{n-1}\right\rangle \\
\left|\lambda_{k, 2 j}^{n-1}\right\rangle_{\mathrm{G}_{n, \mathrm{~B}}} & =U_{\phi_{n, \mathrm{~B}}^{-1}}\left|\lambda_{k, 2 j}^{n-1}\right\rangle .
\end{aligned}
$$

Using this in (52) gives

$$
\begin{aligned}
\left|\lambda_{k, 2 j}^{n}\right\rangle & =U_{\phi_{n, \mathrm{~A}}^{-1}}\left|\lambda_{k, 2 j}^{n-1}\right\rangle+c_{k, 2 j}^{n} U_{\phi_{n, \mathrm{~B}}^{-1}}\left|\lambda_{k, 2 j}^{n-1}\right\rangle \\
\left|\lambda_{k+2^{n-1}, 2 j}^{n}\right\rangle & =U_{\phi_{n, \mathrm{~A}}^{-1}}\left|\lambda_{k, 2 j}^{n-1}\right\rangle+d_{k, 2 j}^{n} U_{\phi_{n, \mathrm{~B}}^{-1}}\left|\lambda_{k, 2 j}^{n-1}\right\rangle .
\end{aligned}
$$


The state $U_{\phi_{n, \mathrm{~A}}^{-1}}\left|\lambda_{k, 2 j}^{n-1}\right\rangle$ has support only in $X_{n, \mathrm{~A}}$ and $U_{\phi_{n, \mathrm{~B}}^{-1}}\left|\lambda_{k, 2 j}^{n-1}\right\rangle$ has support only in $X_{n, \mathrm{~B}}$.

We now use the relation

$$
\mathrm{R}_{n} \circ \phi_{n, \mathrm{~B}}^{-1}=\phi_{n, \mathrm{~A}}^{-1},
$$

which implies that

$$
U_{\mathrm{R}_{n}} U_{\phi_{n, \mathrm{~B}}^{-1}}=U_{\phi_{n, \mathrm{~A}}^{-1}}
$$

(Even though (55) and (56) are written specifically at the band-splitting points, they are valid for all $\alpha \in(1,2]$.) Acting on (54) by $U_{\mathrm{R}_{n}}$ and using (56) we get

$$
\begin{aligned}
\lambda_{k, 2 j}^{n}\left|\lambda_{k, 2 j}^{n}\right\rangle & =U_{\mathrm{R}_{n}} U_{\phi_{n, \mathrm{~A}}^{-1}}\left|\lambda_{k, 2 j}^{n-1}\right\rangle+c_{k, 2 j}^{n} U_{\phi_{n, \mathrm{~A}}^{-1}}\left|\lambda_{k, 2 j}^{n-1}\right\rangle \\
-\lambda_{k, 2 j}^{n}\left|\lambda_{k+2^{n-1}, 2 j}^{n}\right\rangle & =U_{\mathrm{R}_{n}} U_{\phi_{n, \mathrm{~A}}^{-1}}\left|\lambda_{k, 2 j}^{n-1}\right\rangle+d_{k, 2 j}^{n} U_{\phi_{n, \mathrm{~A}}^{-1}}\left|\lambda_{k, 2 j}^{n-1}\right\rangle,
\end{aligned}
$$

where (51) has been used on the lhs of (57b). Since $U_{\mathrm{R}_{n}}$ has the flip property that any function with support only in $X_{n, \mathrm{~B}}$ will go entirely to $X_{n, \mathrm{~A}}$ in one iteration and vice-versa we know that $U_{\mathrm{R}_{n}} U_{\phi_{n, \mathrm{~A}}^{-1}}\left|\lambda_{k, 2 j}^{n-1}\right\rangle$ has support only in $X_{n, \mathrm{~B}}$ and $U_{\phi_{n, \mathrm{~A}}^{-1}}\left|\lambda_{k, 2 j}^{n-1}\right\rangle$ has support only in $X_{n, \mathrm{~A}}$. Multiplying (54a) by $\lambda_{k, 2 j}^{n}$ and (54b) by $-\lambda_{k, 2 j}^{n}$ and identifying the components with support only in $X_{n, \mathrm{~A}}$ with the corresponding components in (57) gives

$$
\begin{aligned}
c_{k, 2 j}^{n} U_{\phi_{n, \mathrm{~A}}^{-1}}\left|\lambda_{k, 2 j}^{n-1}\right\rangle & =\lambda_{k, 2 j}^{n} U_{\phi_{n, \mathrm{~A}}^{-1}}\left|\lambda_{k, 2 j}^{n-1}\right\rangle \\
d_{k, 2 j}^{n} U_{\phi_{n, \mathrm{~A}}^{-1}}\left|\lambda_{k, 2 j}^{n-1}\right\rangle & =-\lambda_{k, 2 j}^{n} U_{\phi_{n, \mathrm{~A}}^{-1}}\left|\lambda_{k, 2 j}^{n-1}\right\rangle,
\end{aligned}
$$

showing that $c_{k, 2 j}^{n}=\lambda_{k, 2 j}^{n}$ and $d_{k, 2 j}^{n}=-\lambda_{k, 2 j}^{n}$. Using this result gives the pair of recursion relations

$$
\begin{aligned}
\left|\lambda_{k, 2 j}^{n}\right\rangle & =\left(U_{\phi_{n, \mathrm{~A}}^{-1}}+\lambda_{k, 2 j}^{n} U_{\phi_{n, \mathrm{~B}}^{-1}}\right)\left|\lambda_{k, 2 j}^{n-1}\right\rangle \\
\left|\lambda_{k+2^{n-1}, 2 j}^{n}\right\rangle & =\left(U_{\phi_{n, \mathrm{~A}}^{-1}}-\lambda_{k, 2 j}^{n} U_{\phi_{n, \mathrm{~B}}^{-1}}\right)\left|\lambda_{k, 2 j}^{n-1}\right\rangle,
\end{aligned}
$$

which express the right states at the $n^{\text {th }}$ bsp in terms of those at the $(n-1)^{\text {th }}$ bsp.

These recursion relations can be solved to write the right states at the $n^{\text {th }}$ bsp in terms of the right states at the $0^{\text {th }}(\alpha=2)$ bsp. For notational convenience we define

$$
\begin{array}{ll}
\widehat{\mathrm{A}}_{i} & \equiv U_{\phi_{i, \mathrm{~A}}^{-1}} \\
\widehat{\mathrm{B}}_{i} & \equiv U_{\phi_{i, \mathrm{~B}}^{-1}} \\
\widehat{\mathrm{B}}_{k, 2 j, i}^{n} & \equiv\left(\lambda_{k, 2 j}^{n}\right)^{i} \widehat{\mathrm{B}}_{i}
\end{array}
$$


Let $\sigma_{i}(i=1,2, \ldots, n)$ be either 0 or 1 and we define $\widehat{\Pi}_{\sigma_{n} \sigma_{n-1} \ldots \sigma_{1}}$ to be an ordered $n$-product of $\widehat{\mathrm{A}}_{i}$ 's and $\widehat{\mathrm{B}}_{k, 2 j, i}^{n}$ 's, where if $\sigma_{i}=1$ then the $i^{\text {th }}$ location in the $n$-product (counting from the right) will be taken by $\widehat{\mathrm{B}}_{k, 2 j, i}^{n}$ and if $\sigma_{i}=0$ then the $i^{\text {th }}$ location will be taken by $\widehat{\mathrm{A}}_{i}$. Solving (59) gives

$$
\left|\lambda_{k, 2 j}^{n}\right\rangle=\sum_{\{\sigma\}} \widehat{\Pi}_{\sigma_{n} \sigma_{n-1} \ldots \sigma_{1}}\left|\lambda_{1,2 j}^{0}\right\rangle .
$$

The sum in (61) is over all possible $\sigma$-strings of 0's and 1's of length $n$ and so consists of $2^{n}$ terms ( $n$-products). The order of the operators in each $n$-product must be strictly observed since the operators involved do not commute.

To illustrate (61) we write it out explicitly for $n=1$ and 2. For $n=1$ (61) gives

$$
\left|\lambda_{k, 2 j}^{1}\right\rangle=\widehat{\mathrm{A}}_{1}\left|\lambda_{1,2 j}^{0}\right\rangle+\widehat{\mathrm{B}}_{k, 2 j, 1}^{1}\left|\lambda_{1,2 j}^{0}\right\rangle
$$

This agrees with the expression (23) (corresponding to $k=1$ and $k=2$ ) we had for the right eigenstates at the first bsp. For $n=2(61)$ gives

$$
\begin{aligned}
\left|\lambda_{k, 2 j}^{2}\right\rangle= & \widehat{\mathrm{A}}_{2} \widehat{\mathrm{A}}_{1}\left|\lambda_{1,2 j}^{0}\right\rangle+\widehat{\mathrm{A}}_{2} \widehat{\mathrm{B}}_{k, 2 j, 1}^{2}\left|\lambda_{1,2 j}^{0}\right\rangle \\
& +\widehat{\mathrm{B}}_{k, 2 j, 2}^{2} \widehat{\mathrm{A}}_{1}\left|\lambda_{1,2 j}^{0}\right\rangle+\widehat{\mathrm{B}}_{k, 2 j, 2}^{2} \widehat{\mathrm{B}}_{k, 2 j, 1}^{2}\left|\lambda_{1,2 j}^{0}\right\rangle .
\end{aligned}
$$

Now we prove by induction that there is a $2^{n} \times 2^{n}$ Jordan block associated with the eigenvalue 0 at the $n^{\text {th }}$ bsp for each odd order $2 j+1$. In section 2 it was shown that this statement is true for the first bsp. Assume that this statement is true at the $(n-1)^{\text {th }}$ bsp. We denote the Jordan vectors as $\left|0_{k, 2 j+1}^{n-1}\right\rangle$ where $k=2,3, \ldots, 2^{n-1}\left(\left|0_{1,2 j+1}^{n-1}\right\rangle\right.$ is the eigenvector of the block). They satisfy

$$
\begin{aligned}
& U_{\mathrm{R}_{n}}\left|0_{k, 2 j+1}^{n-1}\right\rangle=\left|0_{k-1,2 j+1}^{n-1}\right\rangle, \quad k \neq 1 \\
& U_{\mathrm{R}_{n}}\left|0_{1,2 j+1}^{n-1}\right\rangle=0 .
\end{aligned}
$$

Since we are assuming that $U_{\mathrm{R}_{n-1}}$ has $2^{n-1} \times 2^{n-1}$ Jordan blocks, the conjugacies (44) and (46) imply that $\mathrm{G}_{n, \mathrm{~A}}$ and $\mathrm{G}_{n, \mathrm{~B}}$ both have $2^{n-1} \times 2^{n-1}$ Jordan blocks with states given by

$$
\begin{aligned}
\left|0_{k, 2 j+1}^{n}\right\rangle_{\mathrm{G}_{n, A}} & =\widehat{\mathrm{A}}_{n}\left|0_{k, 2 j+1}^{n-1}\right\rangle \\
\left|0_{k, 2 j+1}^{n}\right\rangle_{\mathrm{G}_{n, B}} & =\widehat{\mathrm{B}}_{n}\left|0_{k, 2 j+1}^{n-1}\right\rangle,
\end{aligned}
$$


where $k=1,2, \ldots, 2^{n-1}$. Since $\mathrm{G}_{n}$ on $\Omega_{n}$ has two $2^{n-1} \times 2^{n-1}$ Jordan blocks for each $j$, $\mathrm{R}_{n}$ on $\Omega_{n}$ can either have two $2^{n-1} \times 2^{n-1}$ Jordan blocks for each $j$ or have one $2^{n} \times 2^{n}$ Jordan block for each $j$. The first case implies that $U_{\mathrm{R}_{n}}$ have two null vectors $\left|0_{1,2 j+1}^{n}\right\rangle_{\mathrm{G}_{n, \mathrm{~A}}}$ and $\left|0_{1,2 j+1}^{n}\right\rangle_{\mathrm{G}_{n, \mathrm{~B}}}$ for each $j$. But $U_{\mathrm{R}_{n}}\left|0_{1,2 j+1}^{n}\right\rangle_{\mathrm{G}_{n, \mathrm{~B}}} \neq 0$, since no function with support in $X_{n, \mathrm{~B}}$ can vanish in one iteration under $U_{\mathrm{R}_{n}}$. Therefore $U_{\mathrm{R}_{n}}$ has a $2^{n} \times 2^{n}$ Jordan block for each odd degree $2 j+1$. This completes the proof by induction.

A null state of $U_{\mathrm{R}_{n}}$ has to be a null state of $U_{\mathrm{G}_{n}}$ too. Therefore the null state of $U_{\mathrm{R}_{n}}$ for each $j$ is given by

$$
\left|0_{1,2 j+1}^{n}\right\rangle=\widehat{\mathrm{A}}_{n}\left|0_{1,2 j+1}^{n-1}\right\rangle
$$

We use the relation

$$
\mathrm{R}_{n} \circ \phi_{n, \mathrm{~A}}^{-1}=\phi_{n, \mathrm{~B}}^{-1} \circ \mathrm{R}_{n-1}
$$

which implies that

$$
U_{\mathrm{R}_{n}} \widehat{\mathrm{A}}_{n}=\widehat{\mathrm{B}}_{n} U_{\mathrm{R}_{n-1}} .
$$

Unlike (56), equation (68) is true only at the band-splitting points. Equation (68) can be used to verify that if we act on both sides of (66) by $U_{\mathrm{R}_{n}}$ its rhs reduces to zero.

The right state $\left|0_{1,2 j+1}^{n}\right\rangle_{\mathrm{G}_{n, \mathrm{~B}}}$ is a good candidate for the Jordan state $\left|0_{2,2 j+1}^{n}\right\rangle$ since under one iteration by $U_{\mathrm{R}_{n}}$ it will have support only in $X_{n, \mathrm{~A}}$ and under two iterations of $U_{\mathrm{R}_{n}}$ it will vanish. Tentatively, from $(65 \mathrm{~b})$ we write

$$
\left|0_{2,2 j+1}^{n}\right\rangle=\widehat{\mathrm{B}}_{n}\left|0_{1,2 j+1}^{n-1}\right\rangle
$$

This guess for the Jordan state can be verified by using relation (56). Similarly (68) can be used to show that

$$
\left|0_{3,2 j+1}^{n}\right\rangle=\widehat{\mathrm{A}}_{n}\left|0_{2,2 j+1}^{n-1}\right\rangle .
$$

and equation (56) can be used to show that

$$
\left|0_{4,2 j+1}^{n}\right\rangle=\widehat{\mathrm{B}}_{n}\left|0_{2,2 j+1}^{n-1}\right\rangle
$$




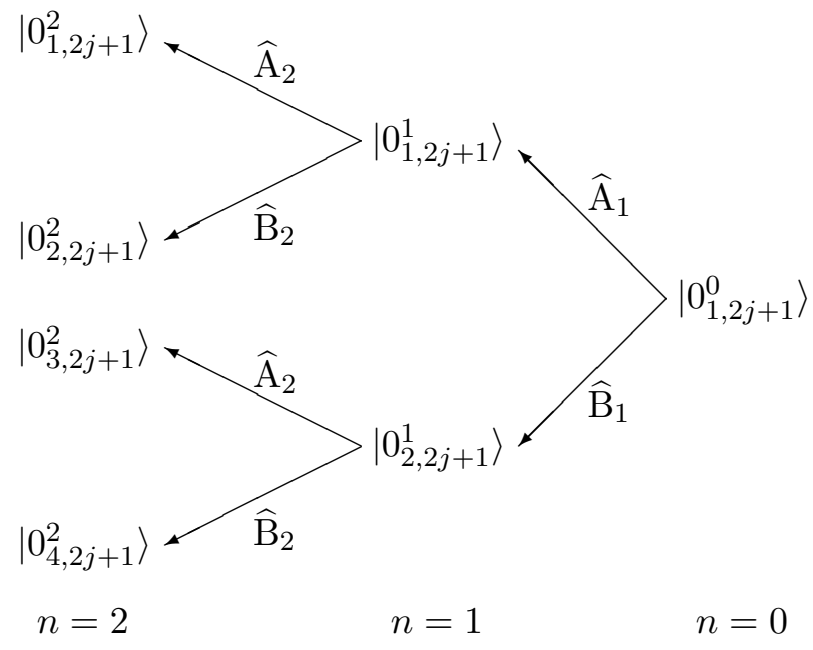

Figure 6: States associated with eigenvalue zero obtained from the action of $\widehat{\mathrm{A}}_{n}$ and $\widehat{\mathrm{B}}_{n}$ on $\left|0_{k, 2 j+1}^{n-1}\right\rangle$.

In general, we find

$$
\begin{array}{ll}
\left|0_{k, 2 j+1}^{n}\right\rangle=\widehat{\mathrm{A}}_{n}\left|0_{\lceil k / 2\rceil, 2 j+1}^{n-1}\right\rangle & \text { for } k \text { odd } \\
\left|0_{k, 2 j+1}^{n}\right\rangle=\widehat{\mathrm{B}}_{n}\left|0_{k / 2,2 j+1}^{n-1}\right\rangle \quad \text { for } k \text { even }
\end{array}
$$

where $k=1,2, \ldots, 2^{n}$ and $\lceil q\rceil$ is the ceiling function ( $q$ if it is an integer or else the next greatest integer). These recursions are illustrated in Figure 6 up to $n=2$. This recursion relation can then be solved to write the eigen/Jordan vectors at the $n^{\text {th }}$ bsp in terms of the null vectors at the $0^{\text {th }} \mathrm{bsp}$. To write down a compact solution we define $\Pi_{\sigma_{n} \sigma_{n-1} \ldots \sigma_{1}}$, which is similar to the $\widehat{\Pi}_{\sigma_{n} \sigma_{n-1} \ldots \sigma_{1}}$ previously defined. We define $\Pi_{\sigma_{n} \sigma_{n-1} \ldots \sigma_{1}}$ to be an ordered $n$-product of $\widehat{\mathrm{A}}_{i}$ 's and $\widehat{\mathrm{B}}_{i}$ 's. If $\sigma_{i}=1$ then the $i^{\text {th }}$ location in the $n$-product (counting from the right) will be taken by $\widehat{\mathrm{B}}_{i}$ and if $\sigma_{i}=0$ then the $i^{\text {th }}$ location will be taken by $\widehat{\mathrm{A}}_{i}$. With each $\Pi_{\sigma_{n} \sigma_{n-1} \ldots \sigma_{1}}$ we associate a binary number formed from the string of 1's and 0's as $\kappa=\sigma_{n} \sigma_{n-1} \ldots \sigma_{1}+1$. Solving the recursion relation (72) we get

$$
\left|0_{\kappa, 2 j+1}^{n}\right\rangle=\Pi_{\sigma_{n} \sigma_{n-1} \ldots \sigma_{1}}\left|0_{1,2 j+1}^{0}\right\rangle,
$$

where $\left|0_{1,2 j+1}^{0}\right\rangle$ is the null vector of degree $2 j+1$ of the tent map with full height, and $\kappa$ here is the decimal equivalent of the binary $\kappa$, which ranges from 1 to $2^{n}$. 


\subsubsection{Left states on the attractor}

We obtain the left states, $\left\langle\lambda_{k, j}^{n}\right|$, which are orthonormal to the right states given by (61) and (73) by taking the duals of those expressions. The dual expression of (61) gives the left states corresponding to the non-zero eigenvalues as

$$
\left\langle\lambda_{k, 2 j}^{n}\right|=\sum_{\{\sigma\}} \widehat{\Pi}_{\sigma_{n} \sigma_{n-1} \ldots \sigma_{1}}^{\dagger} \frac{\left\langle\lambda_{1,2 j}^{0}\right|}{2^{n}},
$$

where the $n$-product here is of $\left(\widehat{\mathrm{A}}_{i}^{-1}\right)^{\dagger}$ 's and $\left(\left(\widehat{\mathrm{B}}_{k, 2 j, i}^{n}\right)^{-1}\right)^{\dagger}$ 's and the factor of $1 / 2^{n}$ is put for normalization. The left states corresponding to the Jordan vectors associated with the eigenvalue 0 are

$$
\left\langle 0_{\kappa, 2 j+1}^{n}\right|=\Pi_{\sigma_{n} \sigma_{n-1} \ldots \sigma_{1}}^{\dagger}\left\langle 0_{1,2 j+1}^{0}\right|,
$$

where $\Pi_{\sigma_{n} \sigma_{n-1} \ldots \sigma_{1}}^{\dagger}$ is an ordered $n$-product of $\left(\widehat{\mathrm{A}}_{i}^{-1}\right)^{\dagger}$ 's and $\left(\widehat{\mathrm{B}}_{i}^{-1}\right)^{\dagger}$ 's.

\subsection{Decay onto the attractor of the rescaled map}

We saw in section 2.1 that $R_{1}$ has no transient bands. The band structure of $G_{2}$ consists of two scaled copies of that of $\mathrm{R}_{1}$ separated by the central interval $X_{2, \mathrm{C}}$. This central interval is a transient band of $R_{2}$. Since $G_{3, A}$ and $G_{3, B}$ have a band structure similar to that of $\mathrm{R}_{2}$ both of them have a transient interval also. We refer to these two transient bands as peripheral transients since in addition there is a central transient in the interval $X_{3, \mathrm{C}}$. In general, as discussed below (49), at the $n^{\text {th }}$ bsp $\mathrm{R}_{n}$ has $2^{n-1}-1$ transient bands, of which $2^{n-1}-2$ are peripheral transient bands. At each bsp all transient bands except the central one are rescaled versions of the central transient bands at previous band-splitting points.

Under the map $\mathrm{R}_{n}$ the inverse image of any point in the central interval $X_{n, \mathrm{C}}$ is contained within the interval itself. This implies that if a function initially has no support in $X_{n, \mathrm{C}}$, it will continue to have no support in $X_{n, \mathrm{C}}$ under repeated iterations by $U_{\mathrm{R}_{n}}$. The spectrum and the form of the eigen/Jordan vector in the central interval at all the band-splitting points can be obtained by inspection. We notice that

$$
U_{\mathrm{R}_{n}}\left[\left(x-x_{n}^{*}\right)^{j} \chi_{n, \mathrm{C}}\right]=(-1)^{j}\left(\frac{1}{\alpha_{n}}\right)^{j+1}\left\{\left(x-x_{n}^{*}\right)^{j} \chi_{n, \mathrm{C}}+\left(x-x_{n}^{*}\right)^{j} \chi_{n, b}\right\},
$$




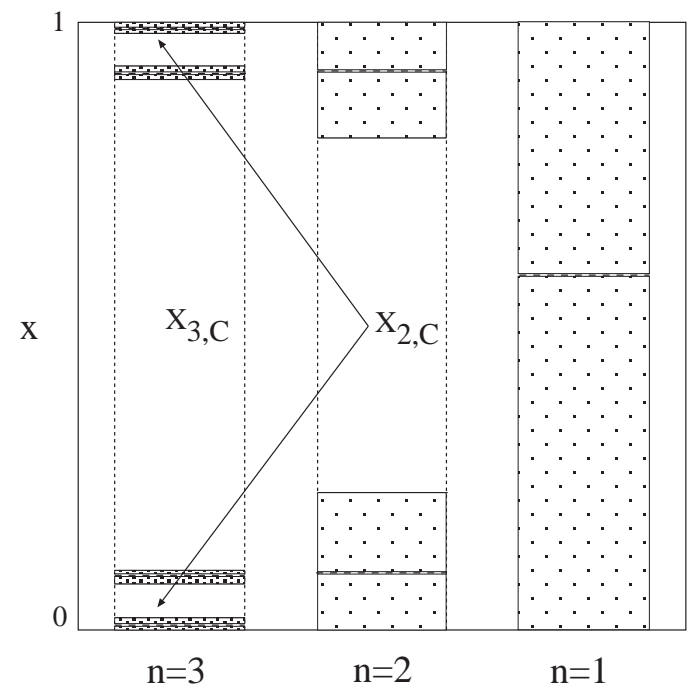

Figure 7: Band structure at the $1^{\text {st }}, 2^{\text {nd }}$ and $3^{\text {rd }}$ band-splitting points. The central transient $X_{2, \mathrm{C}}$ at the $2^{\text {nd }}$ bsp transforms into two peripheral transients at the $3^{\text {rd }}$ bsp.

where $\chi_{n, \mathrm{C}}$ is the indicator function on $X_{n, \mathrm{C}}$ and $\chi_{n, b}=1$ if $x \in\left[\mathrm{R}_{n}^{(3)}\left(x_{c}\right), \mathrm{R}_{n}^{(5)}\left(x_{c}\right)\right)$ and 0 otherwise. The form of the eigen/Jordan vector in $X_{n, \mathrm{C}}$ associated with decay out of the central interval is thus $\left(x-x_{n}^{*}\right)^{j}$. The complete form will be determined below. The eigenvalues associated with decay out of the central transient at the $n^{\text {th }}$ bsp are seen by (76) to be

$$
\phi_{1, j}^{n, 0}=(-1)^{j}\left(\frac{1}{\alpha_{n}}\right)^{j+1}
$$

Next we obtain the complete spectrum at the $n^{\text {th }}$ bsp associated with the decay out of all the transient regions onto $\Omega_{n}$. This is done by transforming all the central transients at band-splitting points of order less than $n$. Of all the peripheral transient bands at the $n^{\text {th }}$ bsp, $2^{n-2}$ of these are $(n-2)$ times rescaled versions of the central transient at $\mathrm{R}_{2}, 2^{n-3}$ of these are $(n-3)$ times rescaled versions of the central transient at $\mathrm{R}_{3}$ and so on up to 2 of these peripheral transients being rescaled versions of the central transient at the $(n-1)^{\text {th }}$ bsp. This is shown in Figure 7 up to $n=3$. We denote the transient eigenvalues at the $n^{\text {th }}$ bsp by $\phi_{k, j}^{n, l}$, where $l$ indicates that it was obtained from the central transient at the 
$(n-l)^{\text {th }}$ bsp, where $l=0,1,2, \ldots, n-2, k$ is an integer from 1 to $2^{l}, j=0,1,2,3, \ldots$, and as before the right state $\left|\phi_{k, j}^{n, l}\right\rangle$ is piecewise-polynomial of degree $j$ in each of the pieces. The eigenvalues are obtained in a similar fashion to (50) as

$$
\phi_{k, j}^{n, l}=\left(\phi_{1, j}^{n-l, 0}\right)^{1 / 2^{l}} \exp \left(\frac{2 \pi i k}{2^{l}}\right)
$$

where the $\phi_{1, j}^{n, 0}$ are given by $(\sqrt{77})$. For even values of $j$ there are degeneracies in the spectrum while for odd values of $j$ there are no degeneracies. We consider first the states associated with the degenerate eigenvalues.

\subsubsection{Transient right states of even degree}

As seen in (78), for a given $l$ (with $n$ and $2 j$ fixed) there are $2^{l}$ distinct eigenvalues indexed by $k$. At each integer step $(l)$ all the eigenvalues of the previous step $(l-1)$ are present and $2^{l-1}$ new eigenvalues appear. But identical eigenvalues from different steps have disparate $k$ values. It is convenient to rearrange the $k$ index so that degenerate eigenvalues share the same $k$. Table 2 contains $\phi_{k, 0}^{5, l}$ for all possible values of $l$ and $k$, arranged to illustrate the reordering in $k$.

\begin{tabular}{|c|cccccccc|}
\hline & $k=1$ & $k=2$ & $k=3$ & $k=4$ & $k=5$ & $k=6$ & $k=7$ & $k=8$ \\
\hline$\phi_{k, 0}^{5,0}$ & $\varphi$ & & & & & & & \\
$\phi_{k, 0}^{5,1}$ & $\varphi$ & $-\varphi$ & & & & & & \\
$\phi_{k, 0}^{5,2}$ & $\varphi$ & $-\varphi$ & $i \varphi$ & $-i \varphi$ & & & & \\
$\phi_{k, 0}^{5,3}$ & $\varphi$ & $-\varphi$ & $i \varphi$ & $-i \varphi$ & $\varphi \exp \left(\frac{\pi i}{4}\right)$ & $\varphi \exp \left(\frac{3 \pi i}{4}\right)$ & $\varphi \exp \left(\frac{5 \pi i}{4}\right)$ & $\varphi \exp \left(\frac{7 \pi i}{4}\right)$ \\
\hline
\end{tabular}

Table 2: Transient spectrum at the $5^{\text {th }}$ bsp with $j=0$. The constant $\varphi \equiv 1 / \alpha_{5}$.

Next we ask if there are independent eigenvectors associated with the degenerate eigenvalues. Using the same procedure used to find the right eigenvectors decaying out of region

\footnotetext{
${ }^{1}$ This can be accomplished by choosing the new $k^{\prime}$ 's asssociated with the $l^{\text {th }}$ step as $k_{2 i-1}^{l}=k_{i}^{l-1}$ and $k_{2 i}^{l}=k_{2 i-1}^{l}+2^{l-1}$, where $i=1, \ldots, 2^{l-1}$ denotes the order of its appearance and $k_{1}^{0}=1$.
} 
I it can be shown that in the rescaled map, for $n>2$ there is no eigenpolynomial of even degree with support in the central transient. This means that the right states associated with $\phi_{1,2 j}^{n, 0}$ are Jordan vectors, for $n>2$. For $n=2$, decay out of the central transient is described by eigenvectors for all values of $j$. Since the right states associated with $\phi_{k, j}^{3,1}, \phi_{k, j}^{4,2}, \ldots, \phi_{k, j}^{n, n-2}$ are transformed versions of $\left|\phi_{1, j}^{2,0}\right\rangle$, these are also eigenvectors. For $n \geq 3$ decay out of the central transient is described by Jordan vectors for even values of $j$. Therefore all the peripheral transients which are related by conjugacies (44) and (46) to these central transients are also Jordan vectors. Hence associated with the transient spectrum are Jordan blocks whose sizes correspond to the algebraic multiplicity of the eigenvalues. Thus from Table 2 we see that at the $5^{\text {th }}$ bsp there is a $4 \times 4$ Jordan block associated with $\phi_{1,2 j}^{5,0}$, a $3 \times 3$ Jordan block associated with $\phi_{2,2 j}^{5,1}$ and so on. At the $n^{\text {th }}$ bsp the largest Jordan block in the transient spectrum is associated with $\phi_{1,2 j}^{n, 0}$ and is of size $n-1 \times n-1$. Since $\phi_{1,0}^{n, 0}$ is the largest eigenvalue with modulus less than 1 in the spectrum of $U_{\mathrm{R}_{\mathrm{n}}}$, it corresponds to the slowest decay mode. Since there is a Jordan block associated with $\phi_{1,0}^{n, 0}$ this decay is modified exponential (polynomial factors in $t$ times exponential decay).

In general (for even $j$ ) we have Jordan vectors for $l \leq n-3$ and eigenvectors for $l=n-2$ as

$$
\begin{aligned}
U_{\mathrm{R}_{n}}\left|\phi_{k, 2 j}^{n, l}\right\rangle & =\phi_{k, 2 j}^{n, l}\left|\phi_{k, 2 j}^{n, l}\right\rangle+\left|\phi_{k, 2 j}^{n, l+1}\right\rangle & & n \geq 3, l \leq n-3 \\
U_{\mathrm{R}_{n}}\left|\phi_{k, 2 j}^{n, n-2}\right\rangle & =\phi_{k, 2 j}^{n, n-2}\left|\phi_{k, 2 j}^{n, n-2}\right\rangle & & n \geq 2 .
\end{aligned}
$$

By inspection we have already obtained the form of the Jordan state in the central region in (76). We have

$$
\left|\phi_{1,2 j}^{n, 0}\right\rangle=a_{n, 2 j}\left(x-x_{n}^{*}\right)^{2 j} \chi_{n, \mathrm{C}}+f_{n, 2 j}(x)
$$

where $f_{n, 2 j}(x)$ is piecewise polynomial with degree $2 j$ over each of the $S_{n}$ intervals (excluding the central interval $X_{n, \mathrm{C}}$ ) at the $n^{\text {th }}$ bsp. Since a Jordan vector multiplied by a scalar doesn't remain a Jordan vector (with respect to the same eigenvector in the block) we do not have the freedom to choose the $a_{n, 2 j}$ 's to be 1 as we did in (36). Using (76) and 
(79) we know that if (80) is to be a Jordan vector the arbitrary functions $f_{n, 2 j}(x)$ must satisfy

$$
U_{\mathrm{R}_{n}} f_{n, 2 j}(x)=\phi_{1,2 j}^{n, 0}\left[f_{n, 2 j}(x)-a_{n, 2 j}\left(x-x_{n}^{*}\right)^{2 j} \chi_{n, b}\right]+\left|\phi_{1,2 j}^{n, 1}\right\rangle
$$

A formal approach to determine the $a_{n, 2 j}$ 's and $f_{n, 2 j}(x)$ is described in Appendix D.

To find the eigenstates corresponding to the peripheral transients we transform the central transient eigenstates at the $2^{\text {nd }}$ bsp to all the higher band-splitting points. We define

$$
\check{\mathrm{B}}_{k, 2 j, i}^{n, n-2}=\left(\phi_{k, 2 j}^{n, n-2}\right)^{i} \widehat{\mathrm{B}}_{i}
$$

and $\check{\Pi}_{\sigma_{n} \sigma_{n-1} \ldots \sigma_{3}}$ is an ordered product of $n-2$ operators $\widehat{\mathrm{A}}_{i}$ and $\check{\mathrm{B}}_{k, 2 j, i}^{n, n-2}(i=3,4, \ldots, n)$. If $\sigma_{i}=0$ the $i^{\text {th }}$ place in the product is taken by $\widehat{\mathrm{A}}_{i}$ and if $\sigma_{i}=1$ the $i^{\text {th }}$ place in the product is taken by $\check{\mathrm{B}}_{k, 2 j, i}^{n, n-2}$. Following the same procedure used to obtain (61) we find that the transient eigenvectors, $\left|\phi_{k, 2 j}^{n, n-2}\right\rangle,(n \geq 3)$, are given by

$$
\left|\phi_{k, 2 j}^{n, n-2}\right\rangle=\sum_{\{\sigma\}} \check{\Pi}_{\sigma_{n} \sigma_{n-1} \ldots \sigma_{3}}\left|\phi_{1, j}^{2,0}\right\rangle
$$

To find the Jordan states corresponding to the peripheral transients, $\left|\phi_{k, 2 j}^{n, l}\right\rangle$ for $1 \leq l \leq$ $n-3$, we use an approach similar to that used to find the Jordan vectors describing decay out of the central transient, $\left|\phi_{1,2 j}^{n, 0}\right\rangle$. To clarify the notation we note that $\left|\phi_{k, 2 j}^{n, 1}\right\rangle$ does not have support in the central transient band, but has support over all the other transient and attracting bands. Similarly $\left|\phi_{k, 2 j}^{n, 2}\right\rangle$ has support on all bands except the central transient band and the two transient bands that are related by a single transformation to the central transient at the $(n-1)^{\text {th }}$ bsp. This pattern continues and $\left|\phi_{k, 2 j}^{n, n-2}\right\rangle$ has support on all the attracting bands and the $2^{n-2}$ transient bands that are related by transformations to the central transient at the $2^{\text {nd }}$ bsp. Following (80) and using the fact that the peripheral transients at the $n^{\text {th }}$ bsp are transformed versions of the transients at the $(n-1)^{\text {th }}$ bsp we write

$$
\left|\phi_{k, 2 j}^{n, l}\right\rangle=a_{k, 2 j}^{n, l}\left(\widehat{\mathrm{A}}_{n}\left|\phi_{\lceil k / 2\rceil, 2 j}^{n-1, l-1}\right\rangle+\phi_{k, 2 j}^{n, l} \widehat{\mathrm{B}}_{n}\left|\phi_{\lceil k / 2\rceil, 2 j}^{n-1, l-1}\right\rangle\right)+g_{k, 2 j}^{n, l}(x)
$$

where $g_{k, 2 j}^{n, l}(x)$ is piecewise polynomial of degree $2 j$. The polynomial $g_{k, 2 j}^{n, l}(x)$ can be obtained using the procedure similar to the one described in Appendix D. We present here 
the solution only for the simplest case,

$$
\left|\phi_{k, 2 j}^{n, n-3}\right\rangle=2 \phi_{k, 2 j}^{n, n-3}\left(\widehat{\mathrm{A}}_{n}\left|\phi_{\lceil k / 2\rceil, 2 j}^{n-1, n-4}\right\rangle+\phi_{k, 2 j}^{n, n-3} \widehat{\mathrm{B}}_{n}\left|\phi_{\lceil k / 2\rceil, 2 j}^{n-1, n-4}\right\rangle\right)-\frac{1}{2 \phi_{k, 2 j}^{n, n-3}}\left|\phi_{k^{\prime}, 2 j}^{n, n-2}\right\rangle
$$

where $k^{\prime}=k+1$ if $k$ is odd and $k^{\prime}=k-1$ if $k$ is even. The recursion relations for the other Jordan vectors involve more terms, and in general to find all the Jordan vectors at the $n^{\text {th }}$ bsp one must know all the Jordan vectors at the $(n-1)^{\text {th }}$ bsp. Also at a particular bsp to determine $\left|\phi_{k, 2 j}^{n, l}\right\rangle$ one must know all the $\left|\phi_{k, 2 j}^{n, l^{\prime}}\right\rangle$ for $l^{\prime}<l$. So generally one proceeds in the following order $\left|\phi_{1,2 j}^{2,0}\right\rangle,\left|\phi_{k, 2 j}^{3,1}\right\rangle,\left|\phi_{1,2 j}^{3,0}\right\rangle,\left|\phi_{k, 2 j}^{4,2}\right\rangle,\left|\phi_{k, 2 j}^{4,1}\right\rangle \ldots$ The transient eigenvectors can be obtained directly from (83) without regard to this order.

\subsubsection{Transient Right states of odd degree}

For odd values of $j$ the transient spectrum at the $n^{\text {th }}$ bsp, given by equation (78), is nondegenerate. We first find the eigenvectors with support in the central transient bands, at all the band-splitting points. By inspection we have already obtained the form of the eigenvector in the central transient in (76) so that

$$
\left|\phi_{1,2 j+1}^{n, 0}\right\rangle=\left(x-x_{n}^{*}\right)^{2 j+1} \chi_{n, C}+f_{n, 2 j+1}(x),
$$

where $f_{n, 2 j+1}(x)$ is a polynomial of degree $2 j+1$ in each of the $S_{n}$ intervals excluding the central interval. For this expression is to be an eigenvector $f_{n, 2 j+1}(x)$ must satisfy

$$
U_{\mathrm{R}_{n}} f_{n, 2 j+1}(x)=\phi_{1,2 j+1}^{n, 0}\left[f_{n, 2 j+1}(x)-\left(x-x_{n}^{*}\right)^{2 j+1} \chi_{n, b}\right] .
$$

This equation for $f_{n, 2 j+1}(x)$ is similar to equation (81), except that it has one term less on the rhs, and may be solved using the procedure outlined in Appendix D.

Once the $\left|\phi_{1,2 j+1}^{n, 0}\right\rangle$ are known the eigenvector corresponding to any $\left|\phi_{k, 2 j+1}^{n, l}\right\rangle$ can be written as

$$
\left|\phi_{k, 2 j+1}^{n, l}\right\rangle=\sum_{\{\sigma\}} \check{\Pi}_{\sigma_{n} \sigma_{n-1} \ldots \sigma_{n-l+1}}\left|\phi_{1,2 j+1}^{n-l, 0}\right\rangle,
$$

where $\check{\Pi}_{\sigma_{n} \sigma_{n-1} \ldots \sigma_{n-l+1}}$ is an ordered product of $l$ operators $\widehat{\mathrm{A}}_{i}$ and $\check{\mathrm{B}}_{k, 2 j, i}^{n, l}(i=n-l+$ $1, \ldots, n)$. The $\check{\mathrm{B}}_{k, 2 j, i}^{n, l}$ operators are defined here as

$$
\check{\mathrm{B}}_{k, 2 j, i}^{n, l} \equiv\left(\phi_{k, 2 j+1}^{n, l}\right)^{i} \widehat{\mathrm{B}}_{i}
$$


If $\sigma_{i}=0$ the $i^{\text {th }}$ place in the product is taken by $\widehat{\mathrm{A}}_{i}$ and if $\sigma_{i}=1$ the $i^{\text {th }}$ place in the product is taken by $\check{\mathrm{B}}_{k, 2 j, i}^{n, l}$. The sum is over all possible strings of 0's and 1's of length $l$.

\subsubsection{Left States}

The left states $\left\langle\phi_{k, j}^{n, l}\right|$ form a bi-orthonormal set with all the previously obtained right states at the $n^{\text {th }}$ bsp as

$$
\begin{aligned}
\left\langle\phi_{k, j}^{n, l} \mid \lambda_{k^{\prime} j^{\prime}}^{n}\right\rangle & =0 \\
\left\langle\phi_{k, j}^{n, l} \mid \phi_{k^{\prime} j^{\prime}}^{n, l^{\prime}}\right\rangle & =\delta_{l l^{\prime}} \delta_{k k^{\prime}} \delta_{j j^{\prime}} .
\end{aligned}
$$

Among the right states only the states with $l=0$ have support in the central transient band. From (80) we see that the associated left states are

$$
\begin{aligned}
\left\langle\phi_{1,2 j}^{n, 0}\right| & =\frac{1}{a_{n, 2 j}} \delta^{(2 j)}\left(x-x_{n}^{*}\right) \\
\left\langle\phi_{1,2 j+1}^{n, 0}\right| & =-\delta^{(2 j+1)}\left(x-x_{n}^{*}\right) .
\end{aligned}
$$

The left states corresponding to the peripheral transients at the $n^{\text {th }} \mathrm{bsp},\left\langle\phi_{k, j}^{n, l}\right|$, are found by transforming the left states at the $(n-1)^{\text {th }} \mathrm{bsp},\left\langle\phi_{\lceil k / 2\rceil, j}^{n-1, l-1}\right|$. Then the entire set at the $n^{\text {th }}$ bsp has to be orthonormalized using a Gram-Schmidt procedure.

\subsection{Back to $\mathrm{T}_{n}(x)$}

Going back to the tent map $\mathrm{T}_{n}(x)$, we transform all the right states of $\mathrm{R}_{n}(x)$ by $U_{\phi_{n}^{-1}}$ and the left states by $K_{\phi_{n}}$. The states describing decay out of $\left[\mathrm{T}_{n}^{(3)}\left(x_{c}\right), 1\right]$ are null states. The eigenvalues describing decay out of $\left[0, \mathrm{~T}_{n}^{(4)}\left(x_{c}\right)\right]$ are $\phi_{j}^{n} \equiv\left(\frac{1}{\alpha_{n}}\right)^{j+1}$ and have associated polynomial eigenvectors of degree $j$. For $j$ even this part of the spectrum overlaps with the spectrum describing decay of transients of $\mathrm{R}_{n}$. There are Jordan vectors associated with this part of the spectrum; denoting them as $\left|\phi_{2 j}^{n}\right\rangle$ we have

$$
\begin{gathered}
U_{\mathrm{T}_{n}}\left|\phi_{2 j}^{n}\right\rangle=\phi_{2 j}^{n}\left|\phi_{2 j}^{n}\right\rangle+\left|\phi_{1,2 j}^{n, 0}\right\rangle \\
U_{\mathrm{T}_{n}}\left|\phi_{2 j+1}^{n}\right\rangle=\phi_{2 j+1}^{n}\left|\phi_{2 j+1}^{n}\right\rangle .
\end{gathered}
$$


These right states can be determined by an extension of the methods used in Section 2.2 to determine the eigenstates describing decay out of interval I for the tent map at the first bsp.

\section{Conclusion}

We have presented the generalized spectral decomoposition of the Frobenius-Perron operator of the tent map at the band-splitting points. The right eigenstates are piecewisepolynomial functions and the left eigenstates are generalized functions. The spectrum is discrete and gives the characteristic decay times of the map. From the decomposition one can calculate correlations of arbitrary polynomials (as well as functions expandable in terms of the polynomial eigenstates). Furthermore, since the modes corresponding to transient decay onto the attractor have been obtained, the full nonequilibrium dynamics of initial probability densities is accessible.

The slowest decay mode, corresponding to the eigenvalue $\alpha_{n}^{-1}$ at the $n^{\text {th }}$ bsp, describes decay onto the attractor. At the $n^{\text {th }}$ bsp there is an $n \times n$ Jordan block associated with this eigenvalue and therefore the decay is modified exponential. The asymptotic periodicity of the map is clearly reflected in the spectrum as at the $n^{\text {th }}$ bsp, all the $n^{\text {th }}$ roots of unity are part of the spectrum. Our analytic solution of density evolution in this system may be useful for comparision with the behavior of systems governed by the Ginzburg-Landau equation since a component of its dynamics [6] can be reduced to the tent map.

\section{Acknowledgements}

We thank I. Prigogine for his support and encouragement and G.E. Ordóñez for several useful discussions and his comments on the manuscript. We acknowledge US Department of Energy grant no. FG03-94ER14465, the Welch Foundation grant no. F-0365 and the European Communities Commision (contract no. 27155.1/BAS) for support of this work. 


\section{Appendix A: Topological conjugacy}

In this appendix we review the spectral decompositions of maps related by a coordinate transformation [4]. Let $\mathrm{T}: X \rightarrow X$ be a map defined on the interval $X$. Transforming the interval $X$ by the one-to-one, onto, continuous function $\phi: X \rightarrow Y$ gives a new map, $\mathrm{S}: Y \rightarrow Y$. This map is determined as

$$
y_{t+1}=\phi\left(x_{t+1}\right)=\phi\left(\mathrm{T}\left(x_{t}\right)\right) \equiv \mathrm{S}\left(y_{t}\right) .
$$

Using that $\phi$ has an inverse gives

$$
\phi\left(\mathrm{T}\left(x_{t}\right)\right)=\phi\left(\mathrm{T}\left(\phi^{-1}\left(\phi\left(x_{t}\right)\right)\right)\right)=\phi \circ \mathrm{T} \circ \phi^{-1}\left(y_{t}\right)
$$

so that

$$
\mathrm{S}=\phi \circ \mathrm{T} \circ \phi^{-1}
$$

The maps $\mathrm{T}$ and $\mathrm{S}$ are said to be topologically conjugate to each other.

The Koopman operator, $K_{\mathrm{S}}$, corresponding to $\mathrm{S}$ is given from (95) by

$$
K_{\mathrm{S}}=K_{\phi}^{-1} K_{\mathrm{T}} K_{\phi},
$$

where we have used the fact that $K_{\phi^{-1}}=K_{\phi}^{-1}$. The Frobenius-Perron operator, $U_{\mathrm{S}}$, coresponding to $\mathrm{S}$ is the adjoint of $K_{\mathrm{S}}$. Taking the adjoint of (96) and using $\left(K_{\phi}^{-1}\right)^{\dagger}=$ $\left(K_{\phi}^{\dagger}\right)^{-1}$ gives

$$
U_{\mathrm{S}}=U_{\phi} U_{\mathrm{T}} U_{\phi}^{-1} \text {. }
$$

Since $U_{\mathrm{S}}$ and $U_{\mathrm{T}}$ are related by the similarity (97) the spectrum of $U_{\mathrm{S}}$ is identical to that of $U_{\mathrm{T}}$ and eigenstates transform as

$$
\left|\lambda_{n}\right\rangle_{\mathrm{S}}=U_{\phi}\left|\lambda_{n}\right\rangle_{\mathrm{T}}
$$

where we use a Dirac-style bra-ket notation for the states. From (96) the left states transform as

$$
\left\langle\left.\lambda_{n}\right|_{\mathrm{S}}=K_{\phi^{-1}}\left\langle\left.\lambda_{n}\right|_{\mathrm{T}} .\right.\right.
$$

Jordan states of the maps are also related as in (98) and (99) and both the algebraic and geometric multiplicities of the eigenvalues are preserved under conjugacy. 


\section{Appendix B: The tent map with unit height}

The Frobenius-Perron operator of the tent map with unit height is given by

$$
U_{\mathrm{T}_{0}} \rho(x)=\frac{1}{2}\left[\rho\left(\frac{x}{2}\right)+\rho\left(\frac{2-x}{2}\right)\right] .
$$

The operator $U_{\mathrm{T}_{0}}$ admits polynomial eigenstates with support on the whole unit interval. Associated with eigenpolynomials of order $2 j$ are the nonzero eigenvalues $2^{-2 j}$. There is an infinite degeneracy of the eigenvalue 0 with an independent odd-order eigenpolynomial associated with each occurence of the eigenvalue. Thus, the odd-order eigenpolynomials are not unique but we choose them as Euler polynomials so that the associated left eigendistributions take a simple form. The right eigenvectors of $U_{\mathrm{T}_{0}}$ are 11, 12

$$
\begin{aligned}
\left|2^{-2 j}\right\rangle_{\mathrm{T}_{0}} & =B_{2 j}(x / 2) \\
\left|0_{2 j+1}\right\rangle_{\mathrm{T}_{0}} & =E_{2 j+1}(x),
\end{aligned}
$$

where $B_{j}(x)$ is the Bernoulli polynomial of order $j$ and $E_{j}(x)$ is the Euler polynomial of order $j$ [14]. The corresponding left states are

$$
\begin{aligned}
\left\langle\left. 2^{-2 j}\right|_{\mathrm{T}_{0}}\right. & =2^{2 j} \widetilde{B}_{2 j}(x) \\
\left\langle\left. 0_{2 j+1}\right|_{\mathrm{T}_{0}}\right. & =\frac{-1}{(2 j+1) !} \delta_{-}^{(2 j+1)}(x-1),
\end{aligned}
$$

where $\tilde{B}_{0}(x)=1$ and for $j \geq 1$

$$
\widetilde{B}_{2 j}(x) \equiv \frac{(-1)^{2 j-1}}{(2 j) !}\left[\delta_{-}^{(2 j-1)}(x-1)-\delta_{+}^{(2 j-1)}(x)\right]
$$

where the action of $\delta_{ \pm}^{(m)}(x-c)$ on a sufficently differentiable function $f(x)$ is given by

$$
\int_{a}^{b} d x \delta_{ \pm}^{(m)}(x-c) f(x)=\lim _{\epsilon \rightarrow 0}(-1)^{m} f^{(m)}(c \pm \epsilon)
$$

for $a \leq c \leq b$ and $\epsilon$ is a positive infinitesimal.

The time evolution of a density is expressed in terms of the spectral decomposition of $U_{\mathrm{T}_{0}}$ as

$$
U_{\mathrm{T}_{0}}^{t} \rho(x)=\sum_{j=0}^{\infty}\left[\left(2^{-2 j}\right)^{t}\left|2^{-2 j}\right\rangle\left\langle 2^{-2 j} \mid \rho\right\rangle+\delta_{t, 0}\left|0_{2 j+1}\right\rangle\left\langle 0_{2 j+1} \mid \rho\right\rangle\right]
$$


where the bilinear form is defined by

$$
\langle f \mid g\rangle \equiv \int_{0}^{1} d x f^{*}(x) g(x) .
$$

\section{Appendix C: Calculation of transient right states}

To determine the functions $g_{\mathrm{II}, j}(x)$ and $g_{\mathrm{III}, j}(x)$, which appear on the rhs of (37) we expand $g_{\mathrm{II}, j}(x) \chi_{\mathrm{II}}+g_{\mathrm{III}, j}(x) \chi_{\mathrm{III}}$ in terms of the eigenstates given in Table 1 of $U_{\mathrm{T}_{1}}$ on the attractor as

$$
\begin{aligned}
g_{\mathrm{II}, j}(x) \chi_{\mathrm{II}}+g_{\mathrm{III}, j}(x) \chi_{\mathrm{III}}= & \sum_{i=1}^{\lfloor j / 2\rfloor} a_{i, j}\left|+2^{-i}\right\rangle_{\Omega}+\sum_{i=1}^{\lfloor j / 2\rfloor} b_{i, j}\left|-2^{-i}\right\rangle_{\Omega} \\
& +\sum_{i=1}^{\left\lfloor\frac{j-1}{2}\right\rfloor} c_{i, j}\left|0_{2 i+1}\right\rangle_{\Omega}+\sum_{i=1}^{\left\lfloor\frac{j-1}{2}\right\rfloor} d_{i, j}\left|0_{J_{2 i+1}}\right\rangle_{\Omega},
\end{aligned}
$$

where $\lfloor x\rfloor$ denotes the integer part (floor) of the real number $x$. Then acting with $U_{\mathrm{T}_{1}}$ gives

$$
\begin{aligned}
U_{\mathrm{T}_{1}}\left(g_{\mathrm{II}, j}(x) \chi_{\mathrm{II}}+g_{\mathrm{III}, j}(x) \chi_{\mathrm{III}}\right)= & \sum_{i=1}^{\lfloor j / 2\rfloor} \frac{a_{i, j}}{2^{i}}\left|+2^{-i}\right\rangle_{\Omega}-\sum_{i=1}^{\lfloor j / 2\rfloor} \frac{b_{i, j}}{2^{i}}\left|-2^{-i}\right\rangle_{\Omega} \\
& +\sum_{i=1}^{\left\lfloor\frac{j-1}{2}\right\rfloor} d_{i, j}\left|0_{2 i+1}\right\rangle_{\Omega} .
\end{aligned}
$$

We substitute (107) and (108) into (37) and act on (37) by all the left states on the attractor in succession. Using orthonormality we obtain the following equations for the expansion coefficients:

$$
\begin{aligned}
\frac{a_{i, j}}{2^{i}}+\alpha_{i, j} & =2^{-(j+1) / 2} a_{i, j} \\
\frac{b_{i, j}}{2^{i}}-\beta_{i, j} & =-2^{-(j+1) / 2} b_{i, j} \\
d_{i, j}+\gamma_{i, j} & =2^{-(j+1) / 2} c_{i, j} \\
d_{i, j} & =0,
\end{aligned}
$$


where

$$
\begin{aligned}
\alpha_{i, j} & \equiv 2^{-(j+1) / 2}\left\langle+2^{-i} \mid x^{j} \chi_{\mathrm{II}}\right\rangle \\
\beta_{i, j} & \equiv 2^{-(j+1) / 2}\left\langle-2^{-i} \mid x^{j} \chi_{\mathrm{II}}\right\rangle=\alpha_{i, j} \\
\gamma_{i, j} & \equiv 2^{-(j+1) / 2}\left\langle 0_{i} \mid x^{j} \chi_{\mathrm{II}}\right\rangle,
\end{aligned}
$$

and we used $\left\langle 0_{J_{i}} \mid x^{j} \chi_{\mathrm{II}}\right\rangle=0$ because $\left\langle 0_{J_{i}}\right|$ has support only in $\chi_{\mathrm{III}}$. Explicit evaluation of $\left\langle+\frac{1}{2^{i}} \mid x^{j} \chi_{\mathrm{II}}\right\rangle$ gives

$$
\left\langle+\frac{1}{2^{i}} \mid x^{j} \chi_{\mathrm{II}}\right\rangle=\left\{\begin{array}{lc}
\frac{\sqrt{2}}{2(\sqrt{2}-1)(j+1)}\left((2-\sqrt{2})^{j}-1\right) & i=0 \\
0 & 2 i-1 \geq j \\
-\frac{j !(2-\sqrt{2})^{j+2 i-1}}{(2 i) !(j-2 i+1) !}\left(2^{(j-2 i+1) / 2}-1\right) & 2 i-1<j .
\end{array}\right.
$$

Evaluation of $\left\langle 0_{i} \mid x^{j} \chi_{\mathrm{II}}\right\rangle$ gives

$$
\left\langle 0_{i} \mid x^{j} \chi_{\mathrm{II}}\right\rangle= \begin{cases}\frac{j !}{(2 i+1) !(j-2 i-1) !}(\sqrt{2}-1)^{j+2 i+1} & 2 i+1 \leq j \\ 0 & \text { otherwise }\end{cases}
$$

These results are then used in (109) to determine the expansion coefficients for the transient eigenstates with support in region I.

\section{Appendix D: Transient right states at higher bsps}

We expand the arbitrary functions $f_{n, 2 j}(x)$ in terms of the transient and non-transient eigenvectors of $U_{\mathrm{R}_{n}}$. The non-transient eigenvectors are given in (61) and (73). The transient eigenvectors will be transformed versions of the central eigenvectors at the previous band-splitting points. The expansion is

$$
\begin{aligned}
f_{n, 2 j}(x)= & \sum_{k=1}^{2^{n}} \sum_{j^{\prime}=1}^{j} b_{k, 2 j^{\prime}}^{n, 2 j}\left|\lambda_{k, 2 j^{\prime}}^{n}\right\rangle+\sum_{k=1}^{2^{n}} \sum_{j^{\prime}=1}^{j-1} c_{k, j^{\prime}}^{n, 2 j}\left|0_{k, 2 j^{\prime}+1}^{n}\right\rangle \\
& +\sum_{l=1}^{n-2} \sum_{k=1}^{2^{l}} \sum_{j^{\prime}=1}^{j} d_{k, j^{\prime}}^{n, l, 2 j}\left|\phi_{k, 2 j^{\prime}}^{n, l}\right\rangle+\sum_{l=1}^{n-2} \sum_{k=1}^{2^{l}} \sum_{j^{\prime}=1}^{j-1} e_{k, j^{\prime}}^{n, l, 2 j}\left|\phi_{k, 2 j^{\prime}+1^{n}}^{n, l}\right\rangle .
\end{aligned}
$$


Since $d_{1, j}^{n, n-2,2 j}$ is the coefficent of the eigenvector of the Jordan block it can be set to zero. Applying $U_{\mathrm{R}_{n}}$ to the function $f_{n, 2 j}(x)$ we get

$$
\begin{aligned}
U_{\mathrm{R}_{n}} f_{n, 2 j}(x) & =\sum_{k=1}^{2^{n}} \sum_{j^{\prime}=1}^{j} b_{k, j^{\prime}}^{n, 2 j} \lambda_{k, 2 j^{\prime}}^{n}\left|\lambda_{k, 2 j^{\prime}}^{n}\right\rangle+\sum_{k=1}^{2^{n}-1} \sum_{j^{\prime}=1}^{j-1} c_{k+1, j^{\prime}}^{n, 2 j}\left|0_{k, 2 j^{\prime}+1}^{n}\right\rangle \\
& +\sum_{l=1}^{n-2} \sum_{k=1}^{2^{l}} \sum_{j^{\prime}=1}^{j} d_{k, j^{\prime}}^{n, l, 2 j} \phi_{k, 2 j^{\prime}}^{n, l}\left|\phi_{k, 2 j^{\prime}}^{n, l}\right\rangle+\sum_{l=2}^{n-2} \sum_{k=1}^{2^{l-1}} \sum_{j^{\prime}=1}^{j} d_{k, j^{\prime}}^{n, l-1,2 j} \phi_{k, 2 j^{\prime}}^{n, l}\left|\phi_{k, 2 j^{\prime}}^{n, l}\right\rangle \\
& +\sum_{l=1}^{n-2} \sum_{k=1}^{2^{l}} \sum_{j^{\prime}=1}^{j} e_{k, j^{\prime}}^{n, l, 2 j} \phi_{k, 2 j^{\prime}+1}^{n, l}\left|\phi_{k, 2 j^{\prime}+1}^{n, l}\right\rangle
\end{aligned}
$$

We substitute (114) and (113) into (81) and hit both sides of the equation with $\left\langle\lambda_{k, 2 j^{\prime}}^{n}\right.$, $\left\langle 0_{k, 2 j^{\prime}+1}^{n}\right|,\left\langle\phi_{k, 2 j^{\prime}+1}^{n, l}\right|$ and $\left\langle\phi_{k, 2 j^{\prime}}^{n, l}\right|$ successively. Letting

$$
\begin{aligned}
\alpha_{k, j^{\prime}}^{n, 2 j} & \equiv\left\langle\lambda_{k, 2 j^{\prime}}^{n} \mid\left(x-x_{n}^{*}\right)^{2} j \chi_{b, n}\right\rangle \\
\beta_{k, j^{\prime}}^{n, 2 j} & \equiv\left\langle 0_{k, 2 j^{\prime}+1}^{n} \mid\left(x-x_{n}^{*}\right)^{2 j} \chi_{b, n}\right\rangle \\
\gamma_{k, j^{\prime}}^{n, l, 2 j} & \equiv\left\langle\phi_{k, 2 j^{\prime}}^{n, l} \mid\left(x-x_{n}^{*}\right)^{2 j} \chi_{b, n}\right\rangle
\end{aligned}
$$

we obtain the following equations for the expansion coefficents $a_{n, j}, b_{k, j^{\prime}}^{n, 2 j}, c_{k, j^{\prime}}^{n, 2 j}, d_{k, j^{\prime}}^{n, l, 2 j}$ and $e_{k, j^{\prime}}^{n, l, 2 j}$

$$
\begin{aligned}
b_{k, j^{\prime}}^{n, 2 j} \lambda_{k, 2 j^{\prime}}^{n} & =\phi_{1, j}^{n, 0}\left(b_{k, j^{\prime}}^{n, 2 j}-\alpha_{k, j^{\prime}}^{n, 2 j}\right) \\
c_{k+1, j^{\prime}}^{n, 2 j} & =\phi_{1, j}^{n, 0}\left(c_{k, j^{\prime}}^{n, 2 j}-\beta_{k, j^{\prime}}^{n, 2 j}\right) \\
a_{n, 2 j} \phi_{1,2 j}^{n, 0} \gamma_{1, j}^{n, 1,2 j} & =1 \\
e_{k, j^{\prime}}^{n, l, 2 j} \phi_{k, 2 j^{\prime}+1}^{n, l} & =\phi_{1, j}^{n, 0}\left(e_{k, j^{\prime}}^{n, l, 2 j}-\delta_{k, j^{\prime}}^{n, l, 2 j}\right) .
\end{aligned}
$$

The equations for $d_{k, j^{\prime}}^{n, l, 2 j}$ differ depending on the values of $l, k$ and $j^{\prime}$. For $k=1, j^{\prime}=j$ and $l=2,3, \ldots, n-2$

$$
\begin{aligned}
d_{1, j}^{n, l-1,2 j} & =a_{n, 2 j} \phi_{1,2 j}^{n, 0} \gamma_{1, j}^{n, l, 2 j} \\
d_{1, j}^{n, n-2,2 j} & =0
\end{aligned}
$$

When $j \neq j^{\prime}, k \neq 1$ and $l=1$ we have

$$
d_{k, j^{\prime}}^{n, 1,2 j}\left(\phi_{1,2 j}^{n, 0}-\phi_{k, 2 j^{\prime}}^{n, 1}\right)=a_{n, j} \phi_{1,2 j}^{n, 0} \gamma_{k, j^{\prime}}^{n, 1,2 j} .
$$


When $j \neq j^{\prime}, k \neq 1$ and $l=2,3, \ldots, n-2$ we have

$$
d_{k, j^{\prime}}^{n, l, 2 j}\left(\phi_{1,2 j}^{n, 0}-\phi_{k, 2 j^{\prime}}^{n, l}\right)-d_{k, j^{\prime}}^{n, l-1,2 j}=a_{n, 2 j} \phi_{1,2 j}^{n, 0} \gamma_{k, j^{\prime}}^{n, l, 2 j}
$$

The equations (116) - (119) for $a_{n, 2 j}, b_{k, j^{\prime}}^{n, 2 j}, c_{k, j^{\prime}}^{n, 2 j} d_{k, j^{\prime}}^{n, l, 2 j}$ and $e_{k, j^{\prime}}^{n, l, 2 j}$ are either uncoupled or are coupled in a simple manner and can be solved explicitly to find the expansion coeffients. Plugging these coefficents into $(113)$ and $(\sqrt{80})$ we get all the Jordan states with support in the central transient, for all $n>2$. For $n=2$ set all the $d_{2, k, j^{\prime}}^{l, j}=0, e_{k, j^{\prime}}^{n, l, 2 j}=0$ and $a_{2, j}=1$ and solve for $b_{n, k, j^{\prime}}^{j}, c_{n, k, j^{\prime}}^{j}$ from (119) to obtain the eigenvectors with support in the central transient at the second bsp.

\section{References}

[1] A. Lasota and M.C. Mackey, Chaos, Fractals and Noise (Springer-Verlag, New York, 1994).

[2] H.H. Hasegawa and W.C. Saphir, Phys. Rev. A, 46, 7401 (1992); P. Gaspard, J. Phys. A, 25, L483 (1992); I. Antoniou and S. Tasaki, Physica A, 190, 303 (1992);

I. Antoniou and S. Tasaki, J. Phys. A, 26,73 (1993).

[3] H.H. Hasegawa and D.J. Driebe, Phys. Rev. E 50, 1781 (1994); P. Gaspard, Chaos, 3, 427 (1993); S. Tasaki, I. Antoniou, and Z. Suchanecki, Chaos, Solitons 83 Fractals, 4, 227 (1993).

[4] D.J. Driebe Fully Chaotic Maps and Broken Time Symmetry (Kluwer Academic Publishers, Dordrecht, to appear).

[5] H.G. Schuster Deterministic Chaos (VCH Publishers, New York, 1988).

[6] H.T. Moon, Phys. Rev. E, 47, 772 (1993); H.T. Moon and M.J. Kim, Int. J. Bifurc. Chaos, 7, 1141 (1997). 
[7] S.J. Shenker and L.P. Kadanoff, J. Phys. A, 14, L23 (1981); T. Yoshida, Prog. Theo. Phys., 72, 895 (1984); R. Brown, C. Grebogi and E. Ott, Phys. Rev. A, 34, 2248 (1986).

[8] H. Shigematsu, H. Mori, T. Yoshida and H. Okamoto, J. Stat. Phys., 30, 649 (1983); T. Yoshida, H. Mori and H. Shigematsu, J. Stat. Phys., 31, 279 (1983).

[9] M. Dörfle, J. Stat. Phys., 40, 93 (1985).

[10] N. Provotas and M.C. Mackey, Physica D, 53, 295 (1991).

[11] D.J. Driebe and G.E. Ordóñez, Phys. Lett. A, 211, 204 (1996); G.E. Ordóñez and D.J. Driebe, J. Stat. Phys., 84, 269 (1996).

[12] R.F. Fox, Chaos, 5, 619 (1995); I. Antoniou and B. Qiao, Phys. Lett. A, 215, 280 (1996).

[13] J. Heidel, Phys. Lett. A, 143, 195 (1990).

[14] M. Abramowitz and I.A. Stegun, eds., Handbook of Mathematical Functions, (Dover Publications, New York, 1972). 